\title{
Mathematical approach for the design configuration of magnetic system with multiple electromagnets
}

\author{
Ruipeng Chen, David Folio, Antoine Ferreira* \\ INSA Centre Val de Loire, Université d'Orléans, PRISME EA 4229, Bourges, France
}

\begin{abstract}
Magnetic actuation techniques and microrobots have attracted great interest since they have potential in biomedicine applications. Interventional techniques have emerged as a tool to handle a wide range of minimally invasive surgeries (MIS). However, current MIS procedures are constrained by the limitation of manual operation by surgeon. Thus, various microrobotic solutions including magnetic navigation systems have been proposed for MIS, which carries many potential benefits such as reduced incision, less intraoperative hemorrhaging and postoperative pain, and faster recovery time. In recent decades, many electromagnetic actuation (EMA) systems have been reported and involved to general surgery. The EMA system allows to generate efficiently magnetic source for microrobot control when its specifications are further investigated and satisfied for the desired application. To precisely manipulate the biomedical microrobot, a key issue still relies on the design of a suitable EMA platform. In this paper, we demonstrate a mathematical approach for the design configuration of magnetic system with multiple electromagnets. Especially, the required magnetic coil number has been investigated where the heading motion control, magnetic force control and their combination control are discussed respectively. The singular cases of control are pre-evaluated by a mathematical analysis of the simulated electromagnetic field. In addition, the placed positions and tilted orientations of the applied electromagnets are investigated for the optimization regarding the six typical configurations of EMA platform with 4, 6 and 8 coils. The various configurations of EMA systems have been comprehensively analyzed. Therefore, with the number of electromagnets and their optimal configuration obtained by the proposed approach, the EMA system can be initially established.
\end{abstract}

Keywords: Electromagnetic actuation system, Magnetic microrobot, Design methodology, Robotic magnetic platform

\section{Introduction}

Minimally invasive surgery (MIS) encompasses surgical techniques that limit the size of incisions needed and so lessen wound healing time, associated pain and risk of infection. MIS procedures have been enabled by the advance of various medical technologies. In particular, several surgical robotics systems have been developed. Such robotic platforms already play a significant role to improve patient care, though it increases surgical preparation, cost and risk of the MIS approach compared with traditional open surgery[1,

\footnotetext{
* Corresponding author

Email addresses: david.folioinsa-cvl.fr (David Folio), antoine.ferreira@insa-cvl.fr (Antoine Ferreira)
}

2, 3, 4, 5, 6, 7, 8, 9, 10, 11. For example, there are the well-known da Vinci surgical assis15 tance robots [5, 7, developed by Intuitive Surgical1, which improves the surgeon technical skills. Moreover, unlike the dependence on using rigid instruments with dexterous distal wrists, it is commonly more attractive to use flexible or adaptive robotic 20 tools that access internal anatomy with few skin incisions [10, 12, 13, 14, 15, 16, 17. Especially, the mechanical parts of existing medical robotic devices are still relatively large and rigid to access and treat major inaccessible parts of the human body (e.g. 25 in robot-assisted surgery). In parallel, the various medical robotics solutions have been developed to improve the acceptance of the use of robotics systems in clinical practices. In the meanwhile, mi- 
crorobotics has also emerged as an attractive tech30 nology to introduce novel microsystems to further reduce trauma, create new diagnosis tools and therapeutic procedures.

Indeed, designing miniaturized and versatile microrobotic systems would allow accessing throughout the whole human body; leading to new procedures down to the cellular level; and offering localized diagnosis and treatment with greater precision and efficiency. For example, untethered microscopic devices, smaller than one millimeter, may 40 navigate within the body for targeted therapies [18, 19, 20, 21. Among the various actuation of microrobots, magnetic actuation is considered to be the most promising method [9, 18, 22, 23, 24, 25, 26, 27, 28, 29, 30, 31, 32, 33, 34. To this aim, nu45 merous electromagnetic actuation (EMA) systems have been proposed to control untethered magnetic microrobots for biomedical applications 18, 35, 36, 37, 38, 39, 40, 41, 42, 43, 44, 45, 46, 47. The development of magnetic microrobotic systems 50 circumvents the need to embed power within the microdevice 18, 19, 20. Magnetic microrobot can be then remotely powered and actuated precisely by the use of external electromagnetic fields. This enables untethered microrobots to assist the surgeon to increase precision and dexterity of the MIS procedure 10, 18, 34, 42.

The paper aims to propose a mathematical approach for the design configuration of magnetic system using multiple electromagnets, that improves

60 MIS procedures since the magnetic manipulation of microrobots is one of the most interesting method to assist researchers in many biomedical applications. To address this issue of designing a proper EMA system for a given application, the investiga65 tion of the appropriate design configuration carried out. The paper is then organized as follows. Section 2 presents the theoretical foundation of electromagnetism including the basic principles and necessary equations. From this basis, the electromag70 netic manipulation of untethered microrobot with the magnetic torque and force control is discussed, and the necessary metrics are defined. In Section 3 . the analysis of the minimum number of electromagnets for an EMA platform is investigated. In partic75 ular, singular cases are pointed out when magnetic field and gradient exhibit some linear dependencies. In Section 4, six typical configurations are simulated and evaluated for the efficient motion control of microrobot. The configuration of EMA system is so optimized with respect to the considered magnetic

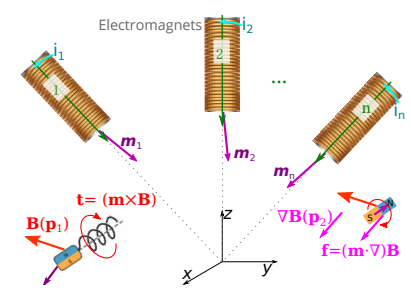

(a)

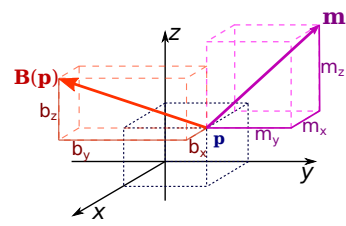

(b)

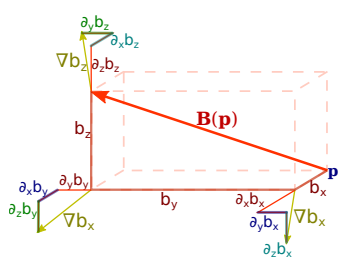

(c)
Figure 1: Electromagnetic manipulation: (a) illustration of the use of the magnetic force and torque on untethered magnetic microrobots; (b) composition of the magnetic field with the magnetic moment of the microrobot; and (c) the different components of the magnetic gradient.

control objectives. In Section 5, the comparison of configurations is performed when the magnetic performances are summarized regarding the arrangement parameters of magnetic system. The paper is concluded with the Section 6 .

\section{Theoretical foundation}

The aim of this section is to recall the basic principles of electromagnetism that serve as a foundation of our research works.

\subsection{Electromagnetic Manipulation}

Starting from the Maxwell's equations, and assuming that charges are either fixed or move as a steady current $J$, the governing equation of a quasistatic magnetic field can be described by the two following relations [4]:

$$
\begin{gathered}
\nabla \cdot B=0 \\
\nabla \times B=\mu_{0} J
\end{gathered}
$$

Next, assuming that the microrobot is a magnetized body described by its magnetic dipole moment $M$, that is placed in a magnetic flux $B$, the induced magnetic force and torque are basically expressed from [49]:

$$
\begin{aligned}
& \mathbf{f}=(M \cdot \nabla) B \\
& \mathbf{t}=(M \times B)
\end{aligned}
$$




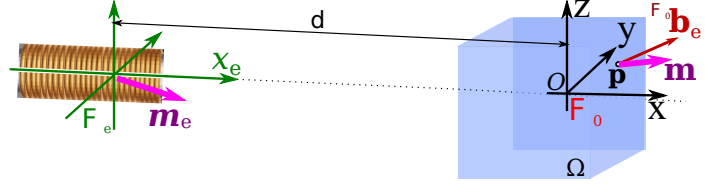

Figure 2: Schematic representation of a single electromagnet inducing a magnetic flux $B_{e}(P)$ within the workspace $\Omega$ (blue square box).

Obviously, $\mathbf{f}$ is related to the magnetic gradient $\nabla B$, whereas the magnetic torque $\mathbf{t}$ is a function of the magnetic field $B$. Moreover, to actuate the microrobot, the magnetic field must undergo either a spatial change (i.e. exhibit a spatial field gradient), or a temporal change, such as through a rotating magnetic field, oscillation and so on. Fig. 1 illustrates these basic principles. Specifically, the magnetic field and its gradient are generated by a set of $n$ electromagnets to control the motion of untethered microrobots. The orientation of microrobot tends to be aligned with the direction of magnetic field $B$, thus the microrobots are rotated by the magnetic torque $\mathbf{t}$. Moreover, the magnetic gradient $\nabla B$ induces the magnetic force $\mathbf{f}$ as propulsion force to move the microrobot. Hence, the magnetic object can be manipulated through the rotation and translation operations using magnetic field and gradient, respectively.

Secondly, if we assume there is no electric current flowing through the workspace occupied by the microrobot, Maxwell's equation implies that equation (2) becomes $\nabla \times B=0$. The magnetic force (3) can be then rearranged using vector calculus into the following form:

$$
\mathbf{f}=\left(\begin{array}{l}
\frac{\partial B}{\partial x} \\
\frac{\partial B}{\partial y} \\
\frac{\partial B}{\partial z}
\end{array}\right) M=\left(\begin{array}{lll}
\frac{\partial b_{x}}{\partial x} & \frac{\partial b_{y}}{\partial x} & \frac{\partial b_{z}}{\partial x} \\
\frac{\partial b_{x}}{\partial y} & \frac{\partial b_{y}}{\partial y} & \frac{\partial b_{z}}{\partial y} \\
\frac{\partial b_{x}}{\partial z} & \frac{\partial b_{y}}{\partial z} & \frac{\partial b_{z}}{\partial z}
\end{array}\right) M=\mathbf{G} M
$$

where $\mathbf{G} \in \mathbb{R}^{3 \times 3}$ denotes the gradient matrix of the magnetic field $B=\left(b_{x}, b_{y}, b_{z}\right)$. Besides, the subscripts $x, y$ and $z$ explicitly refer to the basis directions of the Cartesian reference frame $\mathcal{F}_{0}(O$ : $x, y, z)$ linked to the workspace $\Omega$, in which all vectors are expressed (see Fig. 2).

The torque on microrobot tends to align the magnetization vector with the magnetic field. To rep- 120 resent vector cross products, the skew-symmetric matrix formed of a vector can be employed, that is:

$$
\operatorname{Sk}(M)=\operatorname{Sk}\left(\begin{array}{l}
m_{x} \\
m_{y} \\
m_{z}
\end{array}\right)=\left(\begin{array}{ccc}
0 & -m_{z} & m_{y} \\
m_{z} & 0 & -m_{x} \\
-m_{y} & m_{x} & 0
\end{array}\right)
$$

Therefore, the force (3) and torque (4) applied to magnetic microrobots can be rewritten as:

$$
\left(\begin{array}{l}
\mathbf{f} \\
\mathbf{t}
\end{array}\right)=\left(\begin{array}{c}
(M \cdot \nabla) \\
\operatorname{Sk}(M)
\end{array}\right) B
$$

\subsection{Electromagnetic control}

For multiple-coil configuration, the magnetic field is induced by an EMA system consisting of several electromagnets. Hence, with a given set of $n$ electromagnets, each of them creates a magnetic field, $B_{e}(P)$, at any location of the workspace: $\forall P \in \Omega$. The magnetic fields and their spatial gradients depend linearly on the currents. When $i_{e}$ flows through the coils $e$, the magnetic field can be expressed as: $B_{e}(P)=\widetilde{B}_{e}(P) i_{e}$. The superposition principle is commonly used to compute the overall magnetic field, that is:

$$
\begin{aligned}
B(P) & =\sum_{e=1}^{n} \widetilde{B}_{e}(P) i_{e}=\left(\begin{array}{lllll}
\widetilde{b_{x 1}} & \ldots & \widetilde{b_{x e}} & \ldots & \widetilde{b}_{x n} \\
\widetilde{b_{y}} & \ldots & {\widetilde{b_{y}}}_{y_{e}} & \ldots & {\widetilde{b_{y}}}_{n} \\
\widetilde{b_{z 1}} & \ldots & \widetilde{b}_{z e} & \ldots & \widetilde{b}_{z n}
\end{array}\right) \mathbf{i} \\
& =\left(\begin{array}{l}
\mathcal{B}_{x}(P) \\
\mathcal{B}_{y}(P) \\
\mathcal{B}_{z}(P)
\end{array}\right) \mathbf{i}=\mathcal{B}(P) \mathbf{i}
\end{aligned}
$$

where $\mathbf{i}=\left(i_{1} \ldots i_{n}\right)^{\top}$ is the current vector, and $\mathcal{B}(P)$ is a $3 \times n$ matrix mapping the currents to the magnetic fields. Similarly, the magnetic gradient fields could be expressed as:

$$
\begin{aligned}
& \frac{\partial B(P)}{\partial x}=\sum_{e=1}^{n} \frac{\partial \widetilde{B}_{e}(P)}{\partial x} i_{e}=\mathcal{G}_{x}(P) \mathbf{i} \\
& \frac{\partial B(P)}{\partial y}=\sum_{e=1}^{n} \frac{\partial \widetilde{B}_{e}(P)}{\partial y} i_{e}=\mathcal{G}_{y}(P) \mathbf{i} \\
& \frac{\partial B(P)}{\partial z}=\sum_{e=1}^{n} \frac{\partial \widetilde{B}_{e}(P)}{\partial z} i_{e}=\mathcal{G}_{z}(P) \mathbf{i}
\end{aligned}
$$

where each $\mathcal{G}_{x, y, z}(P)$ is $3 \times n$ matrix mapping the current to the magnetic gradient field in the $x, y$, and $z$ directions, respectively.

The different mapping matrices $\mathcal{B}(P)$ and $\mathcal{G}_{x, y, z}(P)$ expressions are related to the type and 
geometry of each electromagnet (e.g. length, radius, numbers of turns). For instance, the mapping for Helmholtz or Maxwell coils could be easily car- 150 types of electromagnets, especially with a magnetic core, the mapping matrices can be calculated either from measures of the magnetic field within the EMA system's workspace or from the simulations of magnetic field using FEM models 43 .

Finally, the equations of magnetic force and torque can be rearranged as follows:

$$
\begin{aligned}
\left(\begin{array}{l}
\mathbf{t} \\
\mathbf{f}
\end{array}\right) & =\left(\begin{array}{c}
\operatorname{Sk}(M) \mathcal{B} \\
M^{\top} \mathcal{G}_{x}(P) \\
M^{\top} \mathcal{G}_{y}(P) \\
M^{\top} \mathcal{G}_{z}(P)
\end{array}\right) \mathbf{i}=\left(\begin{array}{c}
\mathcal{A}_{t}(M, P) \\
\mathcal{A}_{f}(M, P)
\end{array}\right) \mathbf{i} \\
& =\mathcal{A}(M, P) \mathbf{i}
\end{aligned}
$$

where $\mathcal{A}_{t}(M, P)$ and $\mathcal{A}_{f}(M, P)$ are the actuation matrices mapping the current vector $\mathbf{i}$ to the torque and force, respectively; and $\mathcal{A}(M, P)$ is the $6 \times n$ actuation matrix mapping to the applied magnetic wrench. These magnetic actuation matrices depend both on the position $P \in \Omega$, and on the orientation of the dipole moment $M$ of the microrobot.

According to equations (3)-(4), each column of the matrix $\mathcal{A}(M, P)$ represents the wrench on the force and torque per current unit created by each electromagnet. If there are greater than $n>6$ electromagnets, the actuation matrix $\mathcal{A}(M, P)$ leads to a better conditioned matrix, a more isotropic workspace $\Omega$, a reduction of singularity configurations, and lower current requirements [50, 51]. In such cases, $n>6$, the EMA system could be said "redundant" for the task. Especially, if $\mathcal{A}(M, P)$ is of full rank, for a desired force, $\mathbf{f}^{\star}$ and torque, $\mathbf{t}^{\star}$, the actuation currents $\mathbf{i}$ can be calculated from the pseudo-inverse:

$$
\mathbf{i}=\mathcal{A}^{+}(M, P)\left(\begin{array}{l}
\mathbf{t}^{\star} \\
\mathbf{f}^{\star}
\end{array}\right)
$$

If $n<6$, the pseudo-inverse would be a leastsquares approximations. Hence, for a controlled force and torque, the input current can be obtained only if the pseudo-inverse of $\mathcal{A}(M, P)$ exists. This derivation on the controlled current $\mathbf{i}$ can be similarly extended for controllers that require torque and/or force control 52 .

\subsection{Indexes for Electromagnetic System}

To be able to compare such various electromagnetic coils dispositions, it is necessary to define some metrics to get some quantitative assessments of their effectiveness. The basic idea is to set a score to a given EMA configuration to allow an effective comparative analysis. Indeed, with respect to the applications objectives, different indexes can be defined and considered.

\subsubsection{Magnetic Field Indexes}

First, the strength and homogeneity of either the magnetic field or its gradient in the workspace are the most significant metrics for medical applications. As example, a strong magnetic field strength leads to powerful torque $\mathbf{t}$ enabling reliable swim160 ming of the helical microrobot. In the meantime, it should be homogeneous along the workspace to simplify the control strategy. Furthermore, the EMA system needs to avoid unexpected magnetic force when varying the orientation of microrobot along with the magnetic field. Similarly, a strong magnetic gradient provides an effective propulsion force f, and its uniformity implies a homogeneous net force on microrobots. To this aim, various indexes can be used from statistical analysis of the fields distributions, such as its maximum, mean, RMS, STD, etc. To evaluate the quality of a magnetic field or its gradient, we have chosen to consider mainly the average and the uniformity indexes introduced hereafter.

Average index. In [40, the authors propose to characterize the strength of a sampled vector field $\varphi$ by its average (i.e. the arithmetic mean) basically defined as:

$$
\langle\varphi\rangle=\frac{1}{N} \sum_{P \in \Omega}^{N} \varphi(P)
$$

with $N$ the number of samples, and $P$ the sampling location in the workspace $\Omega$. This arithmetic mean value reflects the strength of a global vector field $\varphi$. It can be utilized to quantify the average of the magnitudes of a magnetic field or its gradient from the corresponding samples, and then to evaluate the strength of the torque or force in the workspace.

Uniformity index. The isotropy index measures and yields an intuitive value for the difference between each considered vector of a vector field $\varphi$ distribution. To evaluate the isotropy, various indexes can be considered, such as the basic minimum-tomaximum ratio, or the coefficient of variation. For a vector field $\varphi$, the uniformity index $\gamma$ is usually 
considered, which is defined as:

$$
\gamma(\varphi)=1-\frac{1}{2 N\langle\varphi\rangle} \sum_{P \in \Omega}^{N}|\varphi(P)-\langle\varphi\rangle|
$$

where the isotropy index $\gamma$ should be commonly bounded between $0 \%$ and $100 \%$. Then, a uniformity index close to $100 \%$ indicates that every sample of the field $\varphi$ is almost identical.

\subsubsection{Magnetic Actuation Indexes}

In the other hand, the EMA system has to actuate a magnetic microrobot in the workspace. Hence, the ability to perform or not certain motion at any location and direction is of prime importance. Basically, if there are some singularities, some motions cannot be achieved, and the DOFs of the microrobot is reduced. For an EMA system, this analysis can be performed through the examination of its magnetic actuation matrix $\mathcal{A}(M, P)$. As described in 12$), \mathcal{A}(M, P)$ allows mapping the input currents $\mathbf{i}$ to the magnetic torque and force that are applied to a microrobot possessing a magnetic moment $M$ at the location $P \in \Omega$. Hence, the actuation matrix $\mathcal{A}(M, P)$ can be seen as a Jacobian matrix. Commonly, the Jacobian matrix of a system is used to characterize the control efficiency. Here, the columns of the actuation matrix $\mathcal{A}(M, P)$ represents the wrench on the magnetic microrobot. As long as $\mathcal{A}(M, P)$ is singularity-free or merely well-conditioned, the direct mapping will exist and full wrench control of the microrobot can be achieved in the workspace. Thus, the structure of $\mathcal{A}(M, P)$ has to be analyzed to determine the "wrench-kinematic" performance of the EMA system. Therefore, the magnetic actuation matrix provides a similar information as a classic Jacobian matrix in robotics.

There are numerous studies on the kinematic perormance indexes of robotic mechanisms [53, 54, 55, 56. Most of proposed metrics were derived from the definition of manipulability index, introduced by 54 .

Manipulability index. From classic robotic kinematics, measuring the manipulability is a wellknown technique for determining the ability to maneuver in workspace. Specifically, manipulability describes the degree to which a robot can freely apply forces and torques in arbitrary directions, and 220 quantifies the ability to perform an action quickly

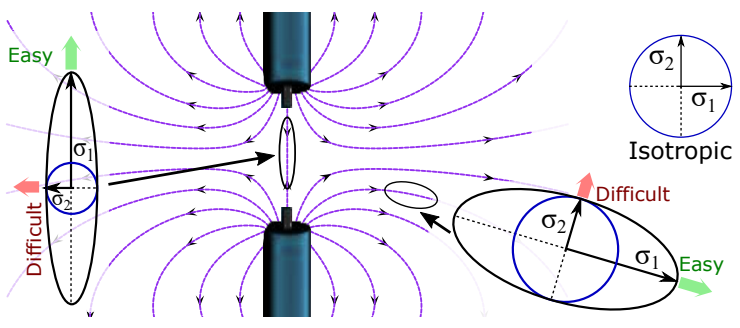

Figure 3: 2D illustration of the manipulability ellipsoid: microrobots have better kinematic capacity in the direction of the major axis $\sigma_{1}$ of the ellipsoid, whereas the minor axis $\sigma_{2}$ induces worse kinematic performance.

and skillfully 54. To do so, the manipulability index is defined as a quality measure describing the distance to singular configurations. The approach is based on analyzing the manipulability ellipsoid that is spanned by the singular vectors of the Jacobian. Similarly, we investigate the mapping efficiency between the current input $\mathbf{i}$ to the torque and force with this standard manipulability ellipsoid. Fig. 3 illustrates the manipulability ellipsoid, here in 2D, where the minor axis $\sigma_{2}$ represents the direction with the worse kinematic capacity, whereas the major axis $\sigma_{1}$ gives the easiest direction of force/torque transmission. Specifically, from the manipulability ellipsoid, the product of the ellipsoid's axes leads to the manipulability index which gives a measure of the maneuverability of the EMA system, and it is defined as 54:

$$
w(\mathcal{A})=\sqrt{\operatorname{det}(\mathcal{A \mathcal { A }} \boldsymbol{\top})}
$$

Commonly, the manipulability ellipsoids can be computed from SVD factorization. Let the SVD of a matrix $\mathcal{A}$ be:

$$
\mathcal{A}=\mathbf{U} \Sigma \mathbf{V}^{\top}
$$

where the $\mathbf{U}$ and $\mathbf{V}$ are orthogonal matrices, with $\mathbf{U}$ a $6 \times 6$ unitary matrix and $\mathbf{V}$ a $n \times n$ unitary matrix. In our considered system, $\Sigma$ is a $6 \times n$ singular value matrix given as follows:

$$
\Sigma=\left(\begin{array}{ccccccccc}
\sigma_{1} & 0 & 0 & 0 & 0 & 0 & 0 & \cdots & 0 \\
0 & \sigma_{2} & 0 & 0 & 0 & 0 & 0 & \cdots & 0 \\
0 & 0 & \sigma_{3} & 0 & 0 & 0 & 0 & \cdots & 0 \\
0 & 0 & 0 & \sigma_{4} & 0 & 0 & 0 & \cdots & 0 \\
0 & 0 & 0 & 0 & \sigma_{5} & 0 & 0 & \cdots & 0 \\
0 & 0 & 0 & 0 & 0 & \sigma_{6} & 0 & \cdots & 0
\end{array}\right)
$$

where $\sigma_{1} \geq \sigma_{2} \geq \ldots \geq \sigma_{6} \geq 0$ are the singular values of $\mathcal{A}$. The singular values are significant indexes for the evaluation of system and that can be 
considered as a scalar gain by which each input is multiplied to produce the corresponding output.

Since the singular values give information about the quality of the workspace [54, the product of 240 all singular values can be used to analyze the control capability of points or configurations in certain directions. Thereby, the measure of manipulability index $w$ can be expressed as the product of the singular values:

$$
w(\mathcal{A})=\prod_{e=1}^{n} \sigma_{e}
$$

It can be noticed that $w$ is proportional to the volume of the manipulability ellipsoid. Generally, a larger measure of $w$ indicates a better conditioning of the actuation matrix $\mathcal{A}(M, P)$, that means an effective control of the microrobot. Hence, the manipulability index $w$ measures the "distance" from singularities, that represents the capability of the EMA system to control efficiently the magnetic microrobot.

Finally, as the above manipulability index 16 245 depends on the scale or units, the normalized manipulability can be preferred:

$$
w_{n}=\frac{w(\mathcal{A}(M, P))}{\max _{P \in \Omega} w(\mathcal{A}(M, P))}
$$

Condition number. Another way to characterize the manipulability ellipsoid is to measure its isotropy. This is commonly achieved by computing the condition number of a matrix, which is defined as:

$$
\kappa(\mathcal{A})=\|\mathcal{A}\|\left\|\mathcal{A}^{\dagger}\right\| \in[1 ;+\infty)
$$

where $\mathcal{A}^{\dagger}$ is the Moore-Penrose pseudo-inverse of $\mathcal{A}$, and $\|$.$\| denotes the Euclidean (or Frobenius) norm$ defined as:

$$
\|\mathcal{A}\|=\sqrt{\operatorname{tr}\left(\mathcal{A W} \mathcal{A}^{\top}\right)}
$$

with $\mathbf{W}$ a weighting matrix, and especially, $\mathbf{W}=\mathbb{I}$ for the Euclidean norm.

To evaluate the control accuracy and isotropy of system, the inverse of the condition number of a matrix, also termed as isotropic index, was introduced by [53, and is defined as:

$$
1 / \kappa=\frac{\sigma_{\min }}{\sigma_{\max }}=\frac{\sigma_{6}}{\sigma_{1}} \in[0 ; 1]
$$

The isotropic index indicates how well the microrobot can move in all directions, and characterizes in some sense the dexterity of the microrobot [56]. Therefore, when $1 / \kappa(\mathcal{A}(M, P))$ is close to 1 , its manipulability ellipsoid is more close to be a sphere, which means the EMA system has more capacity to transmit the same force or torque in all directions (see also Fig. 3).

Let us notice that the previous indexes are only local metrics for a dedicated location $P \in \Omega$. To evaluate the system efficiency over the workspace $\Omega$, many global performance indexes have been proposed [55, 56]. Classically, to define such global performance indexes, the integral of a local performance index $\xi$ over a domain $\mathcal{W}$ is considered given by 24 or its sampled expression 25 :

$$
\begin{aligned}
\Gamma_{\xi}(\mathcal{A}) & =\frac{\int_{\mathcal{W}} \xi(\mathcal{A}) d \mathcal{W}}{\int_{\mathcal{W}} d \mathcal{W}} \\
\Gamma_{\xi}(\mathcal{A}) & =\frac{1}{N} \sum_{x \in \mathcal{W}}^{N} \xi(\mathcal{A}, x)
\end{aligned}
$$

When the domain is the workspace, that is $\mathcal{W}=$ $\Omega \subset \mathbb{R}^{3}$ and $x=P$, and for $\xi=w_{n}, \Gamma_{w_{n}}(\mathcal{A})$ leads to the global manipulability index. Similarly, for $\xi=1 / \kappa, \Gamma_{1 / \kappa}(\mathcal{A})$ denotes the global isotropic index, also termed as the global conditioning index [55, 56.

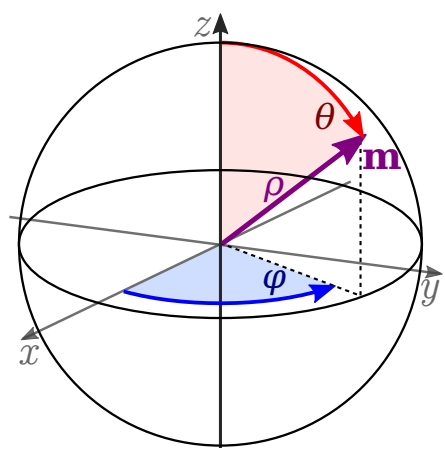

Figure 4: Spherical coordinates $(\rho, \theta, \varphi)$ with $\rho$ the radial distance, $\theta$ the polar angle, and $\varphi$ azimuthal angle.

Likewise, we can evaluate the performance indexes for any direction of the magnetic moment $M$ by considering the $3 \mathrm{D}$ rotation group as domain, leading to: $\mathcal{W}=\mathrm{SO}(3)$, where it means the magnetic moment $M$ has 3D orientations and can be aligned to $x$-, $y$ - and $z$-directions. In the following, to express the orientation of the magnetic moment, 255 we will consider the spherical coordinate representation, as shown in Fig. 4. Obviously, when both location $P \in \Omega$ of the microrobot and the orientation of its magnetic moment $M$ are considered, the domain is defined accordingly, that is $\mathcal{W}=\Omega \times \mathrm{SO}(3)$. 
In this work, the global performance metrics con- 310 sisting of the average index (14), the uniformity index (15), the normalized manipulability index (20), and the (inverse) condition number (23) for EMA systems are proposed. The considered configuration of electromagnets is estimated in relation with these proposed performance metrics. Thereby, the 315 various configurations can be quantitatively evaluated for their magnetic field distribution and actuation efficiency.

\section{DESIGN AND MODELING}

\subsection{The estimation of magnetic coil number}

One of the motivation of an EMA platform is to provide the necessary DOFs for the manipulation of microrobot, mainly depending on the configuration and the number $n$ of constituting electromagnets. Intuitively, it can be shown that platforms with different arrangements of electromagnets exhibit a wide diversity of operating performances for various manipulation tasks [43, 57]. In this section, we will rigorously analyze the number of electromagnets that is required for different magnetic manipulations. Commonly, the manipulation of untethered magnetic microrobot includes the control of its orientation and position. As we mentioned in Fig. 1a the orientation of microrobot can be simply controlled through the magnetic field. In addition, its position is reached using drilling force generated by rotating magnetic field or translating force generated by magnetic gradient. Thus, the efficient remote magnetic manipulation relies on the capability of generating proper magnetic field and gradient. Specifically, the DOFs of wireless microrobot can be evaluated through the magnetic field and its gradient that are generated by the EMA system with a given number of electromagnets.

As demonstrated in 58, 59, 60, 61, at least $n=3$ electromagnets are required to achieve 3 DOFs pointing control. Besides, the position control can be effectively realized with at least $n=4$ magnets in 3D workspace, but up to 5 coils are commonly used to improve the system stability 62 . As computed, 3 DOFs pointing control of the microrobot can be achieved by 2 DOFs torque manipulation. There is no torque that can be applied on the microrobot about its dipole moment main axis. Indeed, some articles have reports several methods to reach 3 DOFs torque control on the designed special microrobots $63,64,65$. For the minimum number of electromagnets, the non-magnetic restoring forces, such as gravity, play also an important role, and will be considered. Moreover, some unexpected singularities involving to force control lead to some holonomic constraints, that will be investigated in the follows.

\subsubsection{Heading motion control}

The quasi-static magnetic fields generated by electromagnets can be defined from the Maxwell's equations (1)-(2). The magnetic torque and force acting on a microrobot are described following (12). In current free space, (1) constraints the gradient matrix of the vector field $B$ to have zero trace; and (2) constraints the gradient matrix of the vector field to be symmetric. Hence, the magnetic force (5) can be rearranged as:

$$
\mathbf{f}=\left(\begin{array}{ccc}
\frac{\partial b_{x}}{\partial x} & \frac{\partial b_{x}}{\partial y} & \frac{\partial b_{x}}{\partial z} \\
\frac{\partial b_{x}}{\partial y} & \frac{\partial b_{y}}{\partial y} & \frac{\partial b_{y}}{\partial z} \\
\frac{\partial b_{x}}{\partial z} & \frac{\partial b_{y}}{\partial z} & -\left(\frac{\partial b_{x}}{\partial x}+\frac{\partial b_{y}}{\partial y}\right)
\end{array}\right)\left(\begin{array}{l}
m_{x} \\
m_{y} \\
m_{z}
\end{array}\right)
$$

This rearrangement allows simplifying the number of magnetic gradient components from nine to five. It is obvious that the magnetic force relies on the applied magnetic gradient field and the magnetic dipole moment of the microrobot. Thus, for a given magnetic dipole, the induced magnetic force only depends on the magnetic gradient that is controlled by the currents flowing through the electromagnets. The magnetic force equations can be thereby expressed as the follows to highlight the magnetic gradients as the controllable parameters:

$$
\begin{aligned}
\mathbf{f} & =\left(\begin{array}{ccccc}
m_{x} & m_{y} & m_{z} & 0 & 0 \\
0 & m_{x} & 0 & m_{y} & m_{z} \\
-m_{z} & 0 & m_{x} & -m_{z} & m_{y}
\end{array}\right)\left(\begin{array}{c}
\frac{\partial b_{x}}{\partial x} \\
\frac{\partial b_{x}}{\partial y} \\
\frac{\partial b_{x}}{\partial z} \\
\frac{\partial b_{y}}{\partial y} \\
\frac{\partial b_{y}}{\partial z}
\end{array}\right) \\
& =\mathcal{F}(M) \mathbf{g}
\end{aligned}
$$

where $\mathcal{F}(M)$ indicates the matrix form of the dipole moment $M$ of the microrobot; and the vector field $\mathrm{g}$ includes the five magnetic gradient components. 
Similarly, the applied magnetic torque is depen- 335 dent on the magnetic field when the dipole moment of the microrobot is determined. Using the skew matrix (6), the Eq. (7) can be rewritten as:

$$
\left(\begin{array}{l}
\mathbf{t} \\
\mathbf{f}
\end{array}\right)=\left(\begin{array}{c}
\mathrm{Sk}(M) \\
\mathcal{F}(M)
\end{array}\right)\left(\begin{array}{l}
B \\
\mathbf{g}
\end{array}\right)
$$

Considering the current flowing through the $n$ electromagnetic coils of the EMA system has a linearly mapping to the magnetic field (8) and gradi- 345 ent $(9)-(11)$, it follows that the 12 can be then represented as:

$$
\begin{aligned}
\left(\begin{array}{l}
\mathbf{t} \\
\mathbf{f}
\end{array}\right) & =\mathcal{A}(M, P) \mathbf{i}=\left(\begin{array}{c}
\mathcal{A}_{t}(M, P) \\
\mathcal{A}_{f}(M, P)
\end{array}\right) \mathbf{i} \\
& =\left(\begin{array}{c}
\operatorname{Sk}(M) \\
\mathcal{F}(M)
\end{array}\right)\left(\begin{array}{l}
\mathcal{B}(P) \\
\mathcal{G}(P)
\end{array}\right) \mathbf{i}
\end{aligned}
$$

where $\mathcal{B}$ is a $3 \times n$ matrix and $\mathcal{G}$ is a $5 \times n$ matrix, that are defined as:

$$
\begin{aligned}
& \mathbf{g}=\mathcal{G}(P) \mathbf{i}
\end{aligned}
$$

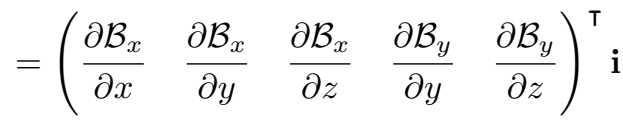

Besides, both $\mathcal{B}$ and $\mathcal{G}$ are a function of the location

$P$ of the microrobot in the workspace $\Omega$. Through investigating the matrices $\mathcal{F}(M)$ and $\operatorname{Sk}(M)$, the conditioning of magnetic field and its gradient can be analyzed.

Since the orientation of microrobot tends to be aligned on the applied magnetic field, it is more convenient to linearize the system by specifying the desired field directly instead of magnetic torque. Hence, the magnetic field and inducing force can be obtained from:

$$
\left(\begin{array}{c}
B \\
\mathbf{f}
\end{array}\right)=\left(\begin{array}{cc}
\mathbb{I} & \mathbb{O} \\
\mathbb{O} & \mathcal{F}(M)
\end{array}\right)\left(\begin{array}{l}
\mathcal{B}(P) \\
\mathcal{G}(P)
\end{array}\right) \mathbf{i}=\mathcal{A}_{b}(M, P) \mathbf{i}
$$

where $\mathbb{I}$ is an identity matrix, and $\mathbb{O}$ is a zero matrix in an appropriately size.

If the microrobot is made of permanent magnet, 355 magnetic torque is linear with the current. If the microrobot is composed of a soft magnetic material and it does not reach the magnetic saturation, there is a linear relation between the magnetic moment and the magnetic field. Consequently, the 360 magnetic torque becomes quadratic with the current 66. Thus, the capability of the EMA system, such as DOFs of manipulation of the microrobot, can be evaluated from the (31). Similarly to the (13), the input currents i can be also here computed using the pseudo-inverse of $\mathcal{A}_{b}(M, P)$ matrix when the matrix has full rank.

Furthermore, as the magnetic field and force are decoupled in (31), they can be first analyzed separately. As the magnetic field is linear with the current $\mathbf{i}$, the sole $B$ field control relies on the properties of the matrix $\mathcal{B}(P)$. It can be easily shown that if the rank of $\mathcal{B}(P)$ is equal to the dimension of the workspace $\forall P \in \Omega$, that leads to a full control of $B$. In other words, the number of current inputs $\mathbf{i}$ should be equal or more than the dimension of the workspace $\Omega$. In contrast, the force control requires more advanced investigations that are presented hereafter.

\subsubsection{Magnetic force control}

The matrix $\mathcal{F}(M)$ is a $3 \times 5$ non-square matrix, and it can be shown that its row rank is full. Thus, $\mathcal{F}(M)$ has a right Moore-Penrose inverse which is expressed by $\mathcal{F}(M)^{\dagger}=\mathcal{F}(M)^{\top}\left(\mathcal{F}(M) \mathcal{F}(M)^{\top}\right)^{-1}$. From (26), the desired magnetic gradient vectors $\mathbf{g}^{\star}$ are thereby obtained using the right inverse when the magnetic force is required. This leads to:

$$
\begin{aligned}
& \left(\begin{array}{c}
\frac{\partial b_{x}^{\star}}{\partial x} \\
\frac{\partial b_{x}^{\star}}{\partial y} \\
\frac{\partial b_{x}^{\star}}{\partial z} \\
\frac{\partial b_{y}^{\star}}{\partial y} \\
\frac{\partial b_{y}^{\star}}{\partial z}
\end{array}\right)=\left(\begin{array}{ccc}
\frac{\left(m_{n}\right) m_{x}}{m_{m}} & \frac{-m_{x}^{2} m_{y}}{m_{m}} & \frac{-m_{z}}{m_{n z}} \\
\frac{m_{y}^{3}+m_{y} m_{z}^{2}}{m_{m}} & \frac{m_{x}^{3}+m_{x} m_{z}^{2}}{m_{m}} & 0 \\
\frac{\left(m_{n}\right) m_{z}}{m_{m}} & \frac{-m_{x} m_{z}^{2}}{m_{m}} & \frac{m_{x}}{m_{n z}} \\
\frac{-m_{x} m_{y}^{2}}{m_{m}} & \frac{\left(m_{n}\right) m_{y}}{m_{m}} & \frac{-m_{z}}{m_{n z}} \\
\frac{-m_{x} m_{z}^{2}}{m_{m}} & \frac{\left(m_{n}\right) m_{z}}{m_{m}} & \frac{m_{y}}{m_{n z}}
\end{array}\right) \mathbf{f} \\
& \mathbf{g}^{\star}=\mathcal{F}(M)^{+} \mathbf{f}
\end{aligned}
$$

where $m_{n}=m_{x}^{2}+m_{y}^{2}+m_{z}^{2}, m_{m}=\left(m_{x}^{2}+m_{y}^{2}+\right.$ $\left.m_{z}^{2}\right)^{2}-\left(m_{x} m_{y}\right)^{2}$, and $m_{n z}=m_{n}+m_{z}^{2}$. As one can see, the matrix $\mathcal{F}(M)$ is a function of the dipole moment $M=\left(m_{x}, m_{y}, m_{z}\right)^{\top}$ of the microrobot. Hence, the pseudo-inverse matrix $\mathcal{F}(M)^{+}$is also affected by the magnetic moment of microrobot.

If the magnetic moment $M$ of the microrobot is fixed (e.g. when a uniform static magnetic field $B_{0}$ is applied), only three independent magnetic gradients of $\mathbf{g}$ are required to produce an arbitrary force f. However, the all five magnetic gradient components are required for continuous force control if $M$ 
is changing. Therefore, there are ten linear rela365

between magnetic gradients.

For instance, linear dependence between $\frac{\partial b_{x}}{\partial x}$ and $\frac{\partial b_{y}}{\partial y}$ is considered. Let us express this linear dependence through adding a coefficient $\alpha \in \mathbb{R}^{*}$, that is: $\frac{\partial b_{y}}{\partial y}=\alpha \frac{\partial b_{x}}{\partial x}$. The Eq. 26 is written with the following $\mathrm{g}$ matrix:

$$
\mathbf{g}=\left(\begin{array}{lllll}
\frac{\partial b_{x}}{\partial x} & \frac{\partial b_{x}}{\partial y} & \frac{\partial b_{x}}{\partial z} & \alpha \cdot \frac{\partial b_{x}}{\partial x} & \frac{\partial b_{y}}{\partial z}
\end{array}\right)^{T}
$$

If the magnetic microrobot is aligned along the $z$ axis, the magnetic force in $z$-axis direction is expressed as:

$$
f_{z}=-(1+\alpha) m_{z} \frac{\partial b_{x}}{\partial x}
$$

Obviously, $f_{z}$ equals to zero when: $\alpha=-1$, that is for $\frac{\partial b_{y}}{\partial y}=-\frac{\partial b_{x}}{\partial x}$.

Let us now assume that the microrobot has a magnetic dipole moment defined by:

$$
M=\left\{(m, \beta m, 0)^{\top},(m, 0, \beta m)^{\top},(0, m, \beta m)^{\top}\right\}
$$

with $\beta \in \mathbb{R}^{*}$ a coefficient term. Then, these values have to be taken into the 32 to investigate the existence of a solution. The necessary and sufficient condition for a matrix reversibility is that the determinant is not equal to 0 . Since the determinant of a matrix is equal to the product of all of their eigenvalues, all eigenvalues must be not equal to 0 in order to enable the existence of the inverse matrix. Thus, the singularly values of the system for the considered linear dependence can be mathematically analyzed by computing whether $\operatorname{det}\left(\mathcal{F}(M) \mathcal{F}(M)^{\top}\right)=0$. The determinants with regard to the magnetic dipole moments of $M$ are obtained as:

$$
\begin{aligned}
\operatorname{det}(m, \beta m, 0) & =m\left(\alpha^{2} \beta^{6}+\alpha^{2} \beta^{4}+2 \alpha \beta^{4}+2 \alpha \beta^{2}\right. \\
& \left.+\beta^{2}+1\right) \\
\operatorname{det}(m, 0, \beta m) & =m\left(\alpha^{2} \beta^{6}+\alpha^{2} \beta^{4}+2 \alpha \beta^{6}+4 \alpha \beta^{4}\right. \\
& \left.+2 \alpha \beta^{2}+\beta^{6}+3 \beta^{4}+3 \beta^{2}+1\right) \\
\operatorname{det}(0, m, \beta m) & =m\left(\alpha^{2} \beta^{6}+3 \alpha^{2} \beta^{4}+3 \alpha^{2} \beta^{2}+\alpha^{2}\right. \\
& \left.+2 \alpha \beta^{6}+4 \alpha \beta^{4}+2 \alpha \beta^{2}+\beta^{6}+\beta^{4}\right)
\end{aligned}
$$

where det represents the determinant operator, such as $\operatorname{det}(M)$ is expressed as the determinant of
When the determinant becomes 0 , the rank of matrix $\mathcal{F}(M)$ is less than 3 and the inverse matrix does not exist, thereby, the system becomes singular. Thus, the system should avoid the relationship between $\beta$ and $\alpha$ regarding the three dipole moments, respectively, meets the followings:

$$
\begin{aligned}
& \beta= \pm \frac{1}{\sqrt{\alpha}} \\
& \beta= \pm \frac{1}{\sqrt{-\alpha-1}} \\
& \beta= \pm \frac{\sqrt{-\alpha(\alpha+1)}}{\alpha+1}
\end{aligned}
$$

When the above equations are satisfied, one of magnetic force components becomes linear dependent to one of the others. For instance, for a dipole moment $M=(m, \beta m, 0)^{\top}$, the basic condition is: $\alpha>0$ and not satisfy 39 , and then a singularity can be avoided. The other singular cases are summarized in Table. (1).

Table 1: The singular cases caused by the linear dependent between $\frac{\partial b_{x}}{\partial x}$ and $\frac{\partial b_{y}}{\partial y}$

\begin{tabular}{|c|l|l|}
\hline$M^{\top}$ & $\begin{array}{l}\text { Relationship } \\
\text { between } \beta \\
\text { and } \alpha\end{array}$ & Condition on $\alpha$ \\
\hline$(m, \beta m, 0)$ & $\beta= \pm \frac{1}{\sqrt{\alpha}}=$ & $\alpha>0$ \\
\hline$(m, 0, \beta m)$ & $\beta \quad 1$ \\
$\pm \frac{1}{\sqrt{-\alpha-1}}$ & $\alpha \neq-1 \wedge \alpha(\alpha+1) \leq 0$ \\
\hline$(0, m, \beta m)$ & $\begin{array}{l}\beta \frac{\sqrt{-\alpha(\alpha+1)}}{\alpha+1} \\
\pm\end{array}$ & \\
\hline
\end{tabular}

Similarly, other nine linearly dependent relations: $\frac{\partial b_{x}}{\partial y}=\alpha \frac{\partial b_{x}}{\partial x}, \frac{\partial b_{x}}{\partial x}=\alpha \frac{\partial b_{x}}{\partial z}, \frac{\partial b_{x}}{\partial x}=\alpha \frac{\partial b_{y}}{\partial z}, \frac{\partial b_{x}}{\partial y}=\alpha \frac{\partial b_{x}}{\partial z}$, $\frac{\partial b_{x}}{\partial y}=\alpha \frac{\partial b_{y}}{\partial y}, \frac{\partial b_{x}}{\partial y}=\alpha \frac{\partial b_{y}}{\partial z}, \frac{\partial b_{x}}{\partial z}=\alpha \frac{\partial b_{y}}{\partial y}, \frac{\partial b_{x}}{\partial y}=\alpha \frac{\partial b_{y}}{\partial z}$, and $\frac{\partial b_{y}}{\partial y}=\alpha \frac{\partial b_{y}}{\partial z}$ have also been investigated. The different conditions to enable a linear relationship between the magnetic gradients terms of $\mathbf{g}$ are summarized hereafter. If these conditions are fulfilled, the matrix $\mathcal{F}(M)$ is not always full rank. Thereby, the magnetic force control becomes singular because the matrix $\mathcal{F}(M)$ is not full rank when any two magnetic gradients exist linear dependence. It means that magnetic force $\mathbf{f}$ cannot be controlled 390 in some orientations and positions where non-full rank happens. To apply an arbitrary force $\mathbf{f}$ on the microrobot in any position $P \in \Omega$ and orientation requires the all five magnetic gradients, whose 
terms must be controlled independently.

It is obvious that the magnetic gradient is a 430 variation in the magnetic field $B$ with respect to position. Thus, when the magnetic gradients are applied on a microrobot for providing propulsion force, it is synchronously placed in a global magnetic field. According to (4), if the magnetic field $B$ is changing, a magnetic torque $\mathbf{t}$ is simultaneously generated on the microrobot, which will change its magnetic moment orientation and then affect the applied magnetic force. For an EMA system to continuously apply a desired force, it must be capa- 440 ble of controlling the microrobot's orientation, and updating the magnetic gradients faster than the change of dipole-moment direction. Therefore, the control of magnetic field should be considered when applying magnetic gradient to the microrobot.

\subsubsection{Combined torque and force control}

Torque control can be analyzed directly through the magnetic field properties, as expressed in (31). Commonly, the three magnetic fields components are required to generate magnetic torque to align microrobot to any directions in the workspace $\Omega$. As discussed above, the control of the five independent magnetic gradients is the necessary and sufficient condition for a singular-free force control. Hence, the (31) can be used to analyze the combined torque and force control. In addition, from equation 31 , the matrix $\mathcal{B}$ and matrix $\mathcal{G}$ are combined as a $6 \times 8$ matrix defined as:

$$
\mathbf{C}(M)=\left(\begin{array}{cc}
\mathbb{I} & \mathbb{O} \\
\mathbb{O} & \mathcal{F}(M)
\end{array}\right)
$$

The first three columns are the expansion of the identity matrix $\mathbb{I}$, thus there is no singular case. The last five columns are relevant to force control. The above matrix $\mathbf{C}(M)$ can be reduced to $\mathcal{C}(M)$ that is a $6 \times 7$ matrix by replacing a linear combination between one of the first three columns and one of the last five columns. The obtained matrix $\mathcal{C}(M)$ describes an EMA system where there is a linear dependence between the magnetic field and its gradient.

Moreover, the all 10 singular cases have been al- ${ }^{450}$ ready investigated regarding linearly dependent relations of magnetic gradients. There is always a singular case if any one of such 10 linear dependencies exists. Besides, there are 3 linear dependencies between magnetic fields. As discussed, these ${ }_{455}$ 3 linear relations will lead to singular case of field control. Thus, only the linear dependence between the first three columns and last five columns should be considered in the analysis of the matrix $\mathbf{C}$. In fact, there are totally 28 linear dependencies in the matrix $\mathbf{C}(M)$.

Consequently, there are 10 dependent relationships between each magnetic gradients, and 3 linear dependencies between the magnetic fields. Since, there is a total of 28 linear dependencies in the matrix $\mathcal{C}$, thereby, the linear combinations between fields and gradients are 15 that will be investigated hereafter.

Let us recall that an unrestrained magnetic microrobot will be aligned with the applied field direction for quasi-static manipulations. Specifically, we assume that the direction of magnetic moment changes slower compared to the rotational time of the alignment of the microrobot with the magnetic field $B$.

Once again, we will discuss linear dependencies on the magnetic gradient component $\frac{\partial b_{x}}{\partial x}$ as examples. These dependencies between $\frac{\partial b_{x}}{\partial x}$ and the magnetic field $B=\left(b_{x}, b_{y}, b_{z}\right)^{\top}$ are defined with the following formulas: a) $\frac{\partial b_{x}}{\partial x}=\alpha b_{x}$, b) $\frac{\partial b_{x}}{\partial x}=\alpha b_{y}$, and c) $\frac{\partial b_{x}}{\partial x}=\alpha b_{z}$, with $\alpha \in \mathbb{R}^{*}$ a coefficient. The corresponding matrices can be expressed respectively as follows:

$$
\begin{aligned}
\mathcal{C}_{a} & =\left(\begin{array}{ccccccc}
1 & 0 & 0 & 0 & 0 & 0 & 0 \\
0 & 1 & 0 & 0 & 0 & 0 & 0 \\
0 & 0 & 1 & 0 & 0 & 0 & 0 \\
\alpha m_{x} & 0 & 0 & m_{y} & m_{z} & 0 & 0 \\
0 & 0 & 0 & m_{x} & 0 & m_{y} & m_{z} \\
-\alpha m_{z} & 0 & 0 & 0 & m_{x} & -m_{z} & m_{y}
\end{array}\right) \\
\mathcal{C}_{b} & =\left(\begin{array}{ccccccc}
1 & 0 & 0 & 0 & 0 & 0 & 0 \\
0 & 1 & 0 & 0 & 0 & 0 & 0 \\
0 & 0 & 1 & 0 & 0 & 0 & 0 \\
0 & \alpha m_{x} & 0 & m_{y} & m_{z} & 0 & 0 \\
0 & 0 & 0 & m_{x} & 0 & m_{y} & m_{z} \\
0 & -\alpha m_{z} & 0 & 0 & m_{x} & -m_{z} & m_{y}
\end{array}\right) \\
\mathcal{C}_{c} & =\left(\begin{array}{ccccccc}
1 & 0 & 0 & 0 & 0 & 0 & 0 \\
0 & 1 & 0 & 0 & 0 & 0 & 0 \\
0 & 0 & 1 & 0 & 0 & 0 & 0 \\
0 & 0 & \alpha m_{x} & m_{y} & m_{z} & 0 & 0 \\
0 & 0 & 0 & m_{x} & 0 & m_{y} & m_{z} \\
0 & 0 & -\alpha m_{z} & 0 & m_{x} & -m_{z} & m_{y}
\end{array}\right)
\end{aligned}
$$

When $\frac{\partial b_{x}}{\partial x}$ and $b_{x}$ are linearly dependent, it is obvious that the matrix $\mathcal{C}_{a}$ is not full rank. The magnetic force on the $x$-direction is directly related to the magnetic field when the dipole moment is $M=\left(m_{x}, 0,0\right)^{\top}$. That is the magnitude of magnetic field will be proportional to the magnetic force required in the dependent direction. However, when the force is applied to a direction, the orientation of the microrobot may be affected due to the 


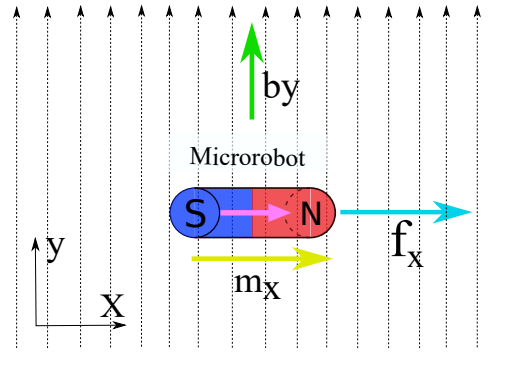

Figure 5: The illustration of the contradiction of microrobot orientation and magnetic field for generating magnetic force.

linear dependence between the generated force and the applied field.

Moreover, there is still an issue on the sign of the force that can not be changed. Here, the magnetic field is used to align the microrobot magnetic moment along the same direction, that is: $m_{x}=\beta b_{x}$, with $\beta \in \mathbb{R}^{*}$ a coefficient. From the above analysis, the $x$-directed magnetic force is not linearly independent to the magnegtic field, which is expressed as: $f_{x}=\alpha b_{x} m_{x}=\alpha \beta b_{x}^{2}$. Hence, the sign of $f_{x}$ can not be changed because the sign of dipole moment and the sign of field are always the same.

Therefore, such control system requires a nonmagnetic restoring force in the $x$-direction. If a suitable nonmagnetic restoring force exists, the EMA system with the linear dependence between $\frac{\partial b_{x}}{\partial x}$ and $b_{x}$ can be used to control the microrobot in the desired direction and position, thus, at least 7 inputs are required.

Some other linearly dependent relationships between magnetic field and its gradient can cause physical impossibility. For instance, a contradiction exists between the orientation of the microrobot 515 and the direction of magnetic field. Indeed, the orientation of microrobot should be aligned along direction of magnetic field as we assumed.

When $\frac{\partial b_{x}}{\partial x}$ and $b_{y}$ are linearly dependent, and if $M=\left(m_{x}, 0,0\right)^{\top}$, it is impossible for any EMA system to apply a magnetic force in the $x$-direction. In this case, the force is expressed by $f_{x}=\alpha b_{y} m_{x}$. Obviously, it is impossible to apply a magnetic force in the $x$-direction . The reason is that it is not feasible to align the microrobot along the $x$-axis when the magnetic field is applied in the $y$-direction. As shown in the Fig. 5 the desired magnetic force requires that the orientation of the microrobot is aligned in $x$-direction that demands the applied magnetic field is aligned to $x$-direction. However, the magnetic field is applied to $y$-direction due to

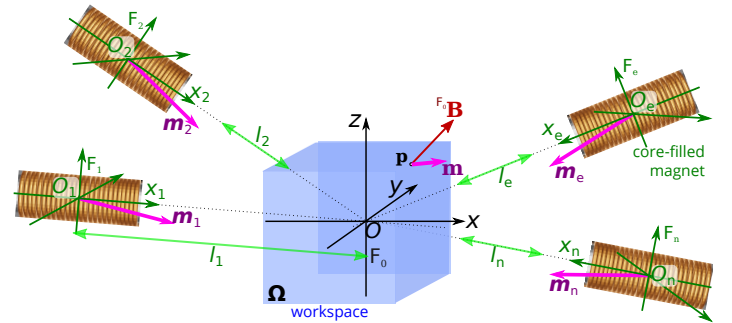

Figure 6: Schematic representation of a multiple electromagnets system inducing a magnetic flux $B(P)$ within the workspace $\Omega$ (blue square box). The length $l_{e}$ represents the distance between the coil center $O_{e}$ to the workspace center $O$.

the assumed linear dependence. Hence, the desired magnetic force can not be produced in respect of such dependent relation. Similarly, when $\frac{\partial b_{x}}{\partial x}$ and $b_{z}$ are in linear dependence, and dipole moment 500 is $M=\left(m_{x}, 0,0\right)^{\top}$, the magnetic force can be expressed as $f_{x}=\alpha b_{z} m_{x}$. This $x$-direction magnetic force can not be generated due to the constraint that the magnetic field aligns the microrobot to applied direction.

Other linearly dependent relations have also analyzed on $\frac{\partial b_{x}}{\partial y}$ : d) $\frac{\partial b_{x}}{\partial y}=\alpha b_{x}$, e) $\frac{\partial b_{x}}{\partial y}=\alpha b_{y}$, and f) $\frac{\partial b_{x}}{\partial y}=\alpha b_{z}$; on $\frac{\partial b_{x}}{\partial z}$ : g) $\frac{\partial b_{x}}{\partial z}=\alpha b_{x}$, h) $\frac{\partial b_{x}}{\partial z}=\alpha b_{y}$, and i) $\frac{\partial b_{x}}{\partial z}=\alpha b_{z}$; on $\frac{\partial b_{y}}{\partial y}$ : j) $\left.\frac{\partial b_{y}}{\partial y}=\alpha b_{x}, \mathrm{k}\right) \frac{\partial b_{y}}{\partial y}=\alpha b_{y}$, and l) $\frac{\partial b_{y}}{\partial y}=\alpha b_{z}$; on $\frac{\partial b_{y}}{\partial z}$ : m) $\left.\frac{\partial b_{y}}{\partial z}=\alpha b_{x}, \mathrm{n}\right)$ $\frac{\partial b_{y}}{\partial z}=\alpha b_{y}$, and p) $\frac{\partial b_{y}}{\partial z}=\alpha b_{z}$. However, the possible linear relations only exist at following cases: $\frac{\partial b_{x}}{\partial x}$ and $\alpha b_{x} ; \frac{\partial b_{y}}{\partial y}$ and $\alpha b_{y}$; or $\frac{\partial b_{x}}{\partial x}+\frac{\partial b_{y}}{\partial y}$ and $\alpha b_{z}$ (as $\left.\frac{\partial b_{x}}{\partial x}+\frac{\partial b_{y}}{\partial y}+\frac{\partial b_{z}}{\partial z}=0\right)$. Such linear dependencies can obviously reduce the inputs for control system that requires less coils. It should be noted that reducing the number of coils only works under some certain conditions, for instance the non-magnetic restoring force is required. In addition, to the study of singularity under the linearly dependencies between input terms, with some advanced control technology application to the magnetic field control, the electromagnetic field singularity problems still can be investigated and avoided 67 .

\subsection{Magnetic field generated in the reference workspace}

Considering the EMA system composed of a set of $n$ electromagnets, the generated magnetic field $\mathcal{F}_{0} B(P)$ at any point $P$ in the workspace $\Omega$ can be illustrated in Fig. 6. This overall magnetic field is assumed to be the sum of the contributions of 
all individual electromagnets. Recall that when the current input $i_{e}$ flows through the coils $e$, the corresponding magnetic field can be expressed as: $B_{e}(P)=\widetilde{B}_{e}(P) i_{e}$. A key step is thus to compute the magnetic field produced by the electromagnets $e$. Several models, based either on numerical or analytical approaches, have been proposed in the literature. Numerical models are commonly based on maps of the magnetic field obtained either from FEM or from experimental measurement of the field $B_{e}$. The numerical method allows a good accuracy but costs a lot of calculation time. Analytical methods are often based on dipole approximation which offers a better computation time, or even on elliptical integrals 68, 69, 70. Hybrid approaches, using a map of the magnetic field obtained from FEM and a fitting of an analytical model can be also considered, such as in 43. The choice of the method commonly leads to make the best trade-off between 5 speed and accuracy. As in this work, numerous simulations are realized, we assume that the magnetic field $B_{e}(P)$ induced by the electromagnet $e$ can be approximated by the magnetic point-dipole model. Specifically, the point-dipole model expresses the ${ }^{55}$ magnetic field ${ }^{\mathcal{F}_{e}} B_{e}(P)$ of the coils $e$ with respect to its own frame $\mathcal{F}_{e}\left(O_{e}: x_{e}, y_{e}, z_{e}\right)$, as shown in Fig. 6, and can be written as:

$$
{ }^{\mathcal{F}_{e}} B_{e}(P)=\frac{\mu_{0}}{4 \pi|P|^{3}}\left(\frac{3\left(M_{e} \cdot P\right)}{|P|^{2}}-M_{e}\right)
$$

where $M_{e}$ is the equivalent magnetic dipole moment related to the magnet source $e$ for a unit current input. Indeed, for the sake of clarity and simplicity, we assume that each electromagnet $e$ can be approximated by its analogous dipole moment $M_{e}$. Hence, the magnetic field is generated by using the point-dipole model for computation. The electromagnet is considered as a theoretical point-dipole mapping to the unit-current contribution, thus the shape or the filled core does not affect the performance analysis of the system. Especially, the manipulability indexes, conditioning indexes and existing singularities are based on the arrangements of electromagnet instead of input current.

The magnetic field ${ }^{\mathcal{F}_{e}} B_{e}(P)$ can be thus expressed in the reference frame $\mathcal{F}_{0}(O: x, y, z)$ linked to the workspace center using the homogeneous transformation:

$$
{ }^{\mathcal{F}_{0}} B_{e}(P)={ }^{\mathcal{F}_{0}} \mathbf{T}_{\mathcal{F}_{e}} \times{ }^{\mathcal{F}_{e}} B_{e}(P)
$$

where the homogeneous transformation matrix is basically defined as:

$$
{ }_{\mathcal{F}_{0}} \mathbf{T}_{\mathcal{F}_{e}}=\left(\begin{array}{c|c}
{ }^{\mathcal{F}_{0}} \mathbf{R}_{\mathcal{F}_{e}} & { }^{\mathcal{F}_{0}} \mathbf{t}_{\mathcal{F}_{e}} \\
\hline \mathbf{0} & 1
\end{array}\right)
$$

where ${ }^{\mathcal{F}_{0}} \mathbf{R}_{\mathcal{F}_{e}}$ and ${ }^{\mathcal{F}_{0}} \mathbf{t}_{\mathcal{F}_{e}}$ denote the rotation and translation matrices with respect to the reference frame $\mathcal{F}_{0}$, respectively.

The overall magnetic field distribution in the workspace produced by a set of $n$ electromagnetic coils can be superposed, that is:

$$
\begin{aligned}
& { }^{\mathcal{F}_{0}} B(P)=\sum_{e=1}^{n}{ }^{\mathcal{F}_{0}} B_{e}(P)=\sum_{e=1}^{n}{ }^{\mathcal{F}_{0}} \tilde{B}_{e}(P) i_{e} \\
& ={ }^{\mathcal{F}}{ }_{0} \mathcal{B}(P) \mathbf{i}=\left({ }^{\mathcal{F}_{0}} \tilde{B}_{1}(P) \quad \ldots \quad{ }^{\mathcal{F}_{0}} \tilde{B}_{n}(P)\right) \mathbf{i}
\end{aligned}
$$

with the input currents $\mathbf{i}=\left(i_{1}, i_{2}, \ldots, i_{n}\right)^{\top}$.

It can be easily shown that the total magnetic field ${ }^{\mathcal{F}_{0}} B(P)$ in the workspace can be changed not only thanks to the currents $\mathbf{i}$, but also by varying the position and/or orientation of electromagnets. If the electromagnets are dynamically moved following a control strategy, a similar current-control approach can be designed.

\section{Configurations of magnetic systems with multiple electromagnets}

The arrangement of electromagnets is also a key issue for the magnetic actuation system. With the number of electromagnets computed by the proposed approach and the derived equations, the simulations of various EMA systems can be performed mathematically. The magnetic characteristics will be investigated according to different configurations of electromagnets with the various performance metrics mentioned in section. 2.3. Thus, in this section, several typical configurations of EMA systems will be quantitatively evaluated.

In a $2 \mathrm{D}$ space, the flat configurations of EMA systems including $n=4,6$ and 8 electromagnets are studied. The 3D-placement setups with $n=6$ and 8 electromagnets are simulated for 3D space. These configurations as illustrated in Fig 7 are selected to estimate the effectiveness of different arrangements of electromagnets.

As shown in Fig 7 a Fig, $7 \mathrm{c}$ the considered fourelectromagnet system, the six-electromagnet system and the eight-electromagnet system are organized in axisymmetric configuration around the center $O$ of the workspace where their coils separated with an offset angle of $90^{\circ}, 60^{\circ}$ and $45^{\circ}$, 


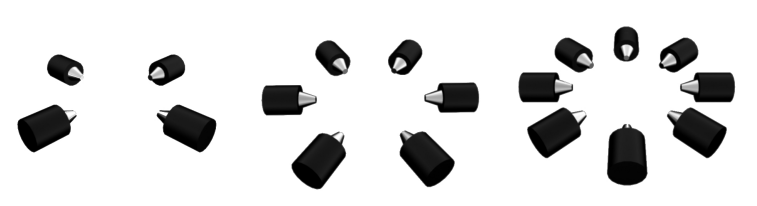

(a)

(b)

(c)

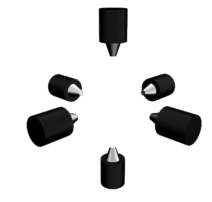

(d)

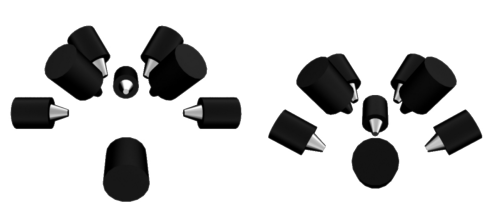

(e)

(f)
Figure 7: Representation of different basic multielectromagnet EMA systems: (a) flat four-electromagnet system; (b) flat six-electromagnet system; (c) flat eightelectromagnet system; (b) 3D six-electromagnet system; (e) OctoMag system (eight coils) and (f) MiniMag system (eight coils).

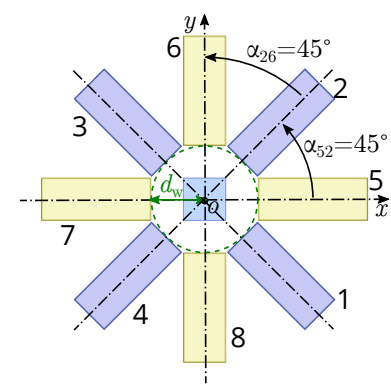

(a)

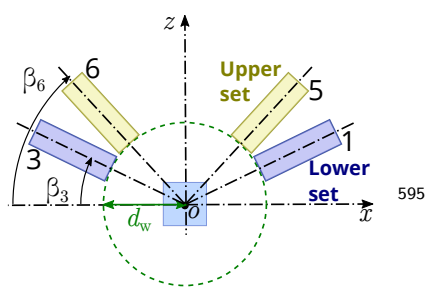

(b) 600
Figure 8: 3D eight coils EMA systems arrangement: (a) top view on $x y$-plane and the (b) side view on $x z$-plane.

respectively. The Fig $7 \mathrm{~d}$ Fig $7 \mathrm{f}$ illustrate the 3Darrangement setups where the 3D six-electromagnet system, eight-electromagnet OctoMag system and eight-electromagnet MiniMag system are represented. The 3D six-electromagnet system consists of three pairs of opposing electromagnets aligned along the $x, y$ and $z$-axis with same distance to the ${ }_{610}$ center $O$. The OctoMag and MiniMag systems designed and developed at the ETH Zurich 43 are both composed of $n=8$ electromagnets. Here, the eight electromagnets have been divided into two sets of four coils referred as the upper and lower 615 sets. As presented in Fig 8a, these two sets are equally spaced and organized around the common $z$-axis of rotation with $\alpha=45^{\circ}$, and are pointing at the center $O$ of the workspace with a fixed distance. For OctoMag system, the lower set is placed in $x y-{ }_{620}$

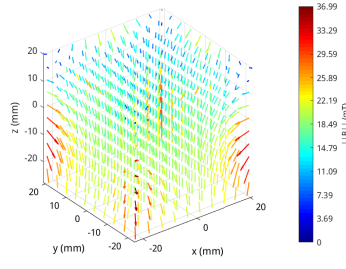

(a)

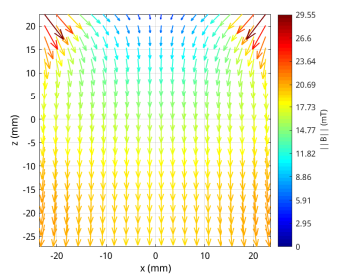

(c)

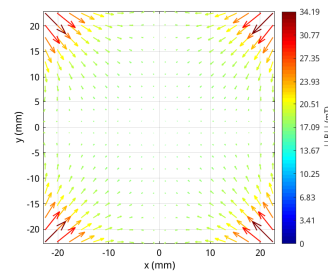

(b)

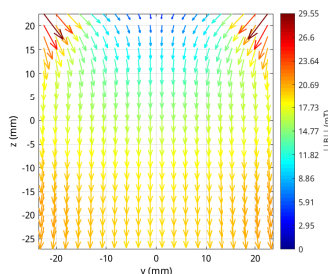

(d)
Figure 9: The magnetic field $B$ generated by OctoMag-like EMA system with $d_{w}=65 \mathrm{~mm}$ : (a) a $3 \mathrm{D}$ view, (b) the $x y$ plane, (c) the $x z$-plane and $(\mathrm{d})$ the $y z$-plane. The colorbar indicates the magnetic field magnitude.

plane, with $\beta_{e}=0(e=1 . .4)$ and the upper are tilted $\beta_{e}=45^{\circ}(e=5 . .8)$. Whereas, the lower set of MiniMag is rotated to $\beta_{e}=26^{\circ}(e=1 . .4)$ from the $x y$-plane, and the upper set is set to $\beta_{e}=47.5^{\circ}$ $(e=5 . .8)$.

In our study, the influence of the distance $d_{w}$ bethe workspace center $O$ and each electromagnet regarding the performance of EMA system is assessed. Also, the impact of tilted angle of the placed electromagnets is evaluated as well to optimize the system performances.

\subsection{Optimization regarding working distance $d_{w}$ of electromagnets for EMA System}

We consider that each coil has the equal distance $d_{w}$ for the considered system as represented in Fig. 8. Different arrangements of EMA system have been investigated from numerous simulations, and different metrics have been calculated to evaluate their performances. To sum up, the values of root mean square (RMS) and uniformity indexes of the magnetic field are synthesized in Table.2, and in Table 3 for its gradient.

Commonly, long distance $d_{w}$ from the workspace center $O$ to the electromagnet leads globally to a slight more uniform magnetic field and gradient, in addition, the effective control of magnetic microrobot is improved. Nevertheless, stronger magnetic 
Table 2: Metrics of the magnetic field strength: its RMS (mT) and uniformity $\gamma(B)$ metric $(\%)$

\begin{tabular}{|l||c|c|c|c|c|}
\hline \multicolumn{1}{|c||}{} & \multicolumn{5}{c|}{ Distance $d_{w}(\mathrm{~mm})$} \\
EMA System & 60 & 65 & 70 & 75 & 80 \\
\hline \hline Flat four-coil (Fig. 7ad & $13.13(71.18)$ & $9.39(70.97)$ & $6.91(70.82)$ & $5.21(70.81)$ & $4.00(70.88)$ \\
Flat six-coil (Fig.7b) & $20.55(72.55)$ & $14.69(72.96)$ & $10.80(73.03)$ & $8.13(72.96)$ & $6.24(72.85)$ \\
Flat eight-coil (Fig. 7c) & $27.41(73.05)$ & $19.59(73.26)$ & $14.41(73.23)$ & $10.85(73.09)$ & $8.33(72.92)$ \\
\hline 3D six-coil (Fig.7d) & $10.08(51.09)$ & $6.48(50.00)$ & $4.28(49.11)$ & $2.90(48.39)$ & $2.01(47.80)$ \\
OctoMag (Fig.7e) & $24.86(76.97)$ & $18.61(79.32)$ & $14.49(81.32)$ & $11.58(82.92)$ & $9.44(84.19)$ \\
MiniMag (Fig.7f) & $55.73(63.86)$ & $42.40(67.20)$ & $33.21(69.89)$ & $26.57(72.12)$ & $21.63(74.02)$ \\
\hline
\end{tabular}

Table 3: Metrics of the magnetic field gradient strength: its RMS (mT/m) and uniformity $\gamma(B)$ metric $(\%)$

\begin{tabular}{|l||c|c|c|c|c|}
\hline \multicolumn{1}{|c|}{} & \multicolumn{5}{c|}{ Distance $d_{w}(\mathrm{~mm})$} \\
EMA System & 60 & 65 & 70 & 75 & 80 \\
\hline \hline Flat four-coil (Fig. 7a & $1.18(59.89)$ & $0.78(64.58)$ & $0.54(68.95)$ & $0.38(72.99)$ & $0.28(76.78)$ \\
Flat six-coil (Fig. 7b) & $1.48(64.27)$ & $0.97(70.66)$ & $0.68(75.34)$ & $0.50(78.69)$ & $0.38(81.21)$ \\
Flat eight-coil (Fig. 7c) & $1.87(66.49)$ & $1.27(71.77)$ & $0.91(75.60)$ & $0.67(78.66)$ & $0.51(81.20)$ \\
\hline 3D six-coil (Fig. 7d) & $1.32(65.61)$ & $0.84(66.97)$ & $0.55(67.50)$ & $0.37(66.94)$ & $0.26(66.42)$ \\
OctoMag (Fig. 7e) & $1.80(36.68)$ & $1.00(43.02)$ & $0.59(49.00)$ & $0.37(54.40)$ & $0.25(59.26)$ \\
MiniMag (Fig.7f) & $2.17(56.69)$ & $1.31(66.02)$ & $0.88(72.13)$ & $0.63(76.26)$ & $0.47(79.18)$ \\
\hline
\end{tabular}

field and gradient require a short length $d_{w}$. Thus, a compromise should be made with respect to the biomedical applications specifications. Certainly, the value of $d_{w}$ will affect the size of the workspace 645 mension.

Obviously, EMA setup with $n=8$ coils produces stronger magnetic field and gradient than configuration with fewer magnets. Furthermore, as pre- 650 in the results of tables, MiniMag arrangement generates the strongest and the most uniform magnetic field, specially in $x y$-plane. From these results, it confirms that the performances of EMA systems are not only affected by the number of coils 655 5 but also by their configurations.

In order to better present the effect of the shifted distance $d_{w}$ of electromagnet, let us focus on the EMA system with a certain number of coils. As mentioned in section 3.1 , the $n=8$ electromag- 660 nets can be applied for the combined force and torque singular-free control with 5 DOFs (3-DOF position and 2-DOF pointing orientation) in a 3D workspace. In the following, we only keep one variable parameter. We have chosen to set the tilted angle similar to the OctoMag EMA setup 43, while working distances of $d_{w}=60 \mathrm{~mm}, 70 \mathrm{~mm}$ and $80 \mathrm{~mm}$ are evaluated, respectively.

As presented in Fig. 9, the magnetic field $B$ distribution is mainly oriented along the $z$-axis direction. As the OctoMag setup shares the same arrangement of electromagnets in the $x y$-plane with the flat four-electromagnet configuration, the vector field in the $x y$-plane is quite homogeneous. The directions of the magnetic field vectors mainly along the $z$-axis are basically due to the upper set of electromagnets.

Fig. 10 shows the magnetic field magnitude $\|B\|$ in the $x y$-plane for the different working distances. The magnetic field distribution $B$ looks much less like a radial field and becomes stronger for the shorter distance $d_{w}$. However, if a magnetic microrobot moves outward from the center of $x y$-plane, 


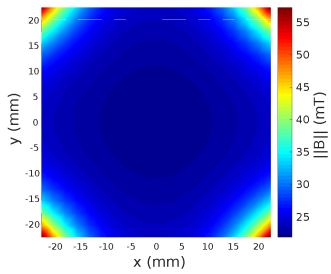

(a)

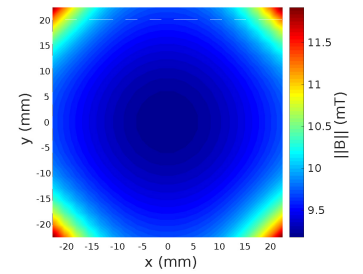

(b)
Figure 10: The magnetic field magnitude $\|B\|$ in the $x y$ plane for lengths of (a) $d_{w}=60 \mathrm{~mm}$, and (b) $d_{w}=80 \mathrm{~mm}$.

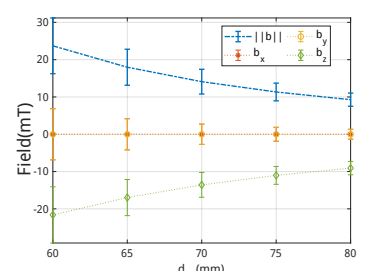

(a)

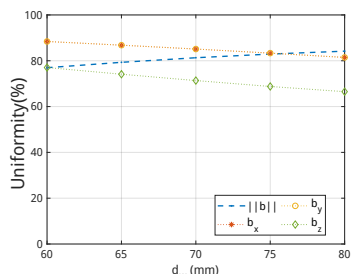

(b)
Figure 11: Magnetic field metrics over the workspace $\Omega$ for $d_{w}$ ranging from $60 \mathrm{~mm}$ to $80 \mathrm{~mm}$ : (a) error-bar showing the average and STD values; and (b) the uniformity index $\gamma$.

the longer distance $d_{w}$ can make magnetic field more homogeneous in each direction. This means that OctoMag system is able to actuate a magnetic microrobot to any direction with less interference in long distances $d_{w}$. For instance, when the working distance is set to $d_{w}=65 \mathrm{~mm}$, the maximum magnetic intensity decreases from $36.99 \mathrm{mT}$ with the $2 \mathrm{D}$ configuration to $35.86 \mathrm{mT}$ with the OctoMag platform.

The magnetic field metrics are shown in Fig. 11 It appears that the average value $\langle\|B\|\rangle$ of the magnetic field magnitude is equivalent to the flat eight-

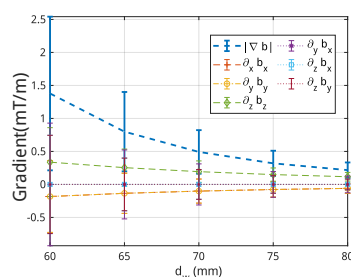

(a)

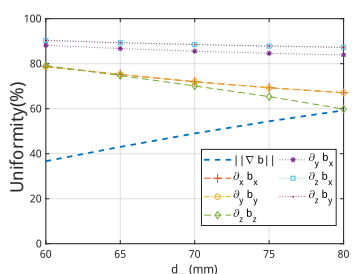

(b)
Figure 12: Magnetic field gradient metrics for distance $d_{w}$ ranging from $60 \mathrm{~mm}$ to $80 \mathrm{~mm}$ : (a) the mean and STD values; and (b) the uniformity index $\gamma$.

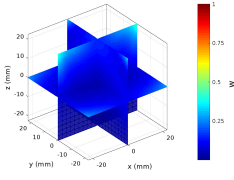

(a)

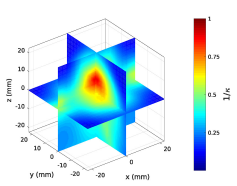

(d)

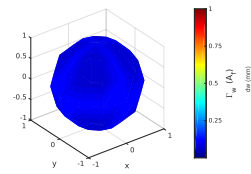

(b)

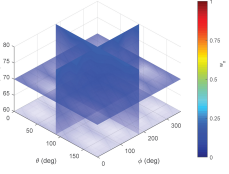

(c)
Figure 13: Performance metrics of the force actuation matrix $\mathcal{A}_{\mathrm{f}}$ : (a) the manipulability $w_{n}$ and (d) conditioning number $1 / \kappa$ indexes for $P \in \Omega$ when $M$ is aligned along the $x$-direction; $(\mathrm{b}) \Gamma_{w_{n}}\left(\mathcal{A}_{\mathbf{f}}\right)$ and $(\mathrm{e}) \Gamma_{1 / \kappa}\left(\mathcal{A}_{\mathbf{f}}\right)$ for the sampled orientation of $M$; and $(\mathrm{c}) \Gamma_{w_{n}}\left(\mathcal{A}_{\mathbf{f}}\right)$ and $(\mathrm{f}) \Gamma_{1 / \kappa}\left(\mathcal{A}_{\mathbf{f}}\right)$ as function of $d_{w}$.

electromagnet arrangement, however, its STD value is here improved. Furthermore, the uniformity in$\operatorname{dex} \gamma(\|B\|)$ is more important and is increasing with the length $d_{w}$. Therefore, the values confirm that OctoMag-like arrangement is a promising solution to efficiently actuate a magnetic microrobot in a $3 \mathrm{D}$ workspace.

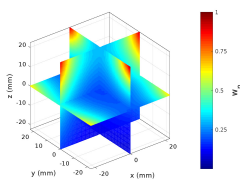

(a)

(d)

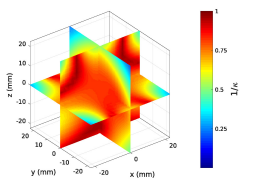

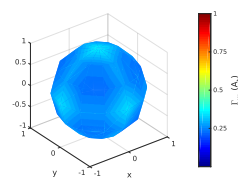

(b)

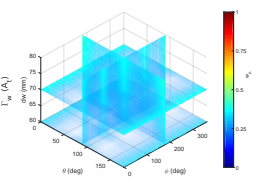

(c)

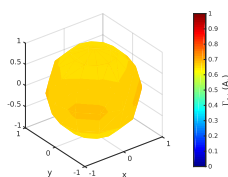

(e)

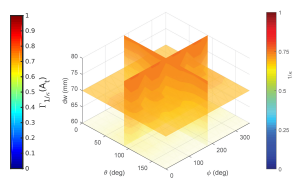

(f)
Figure 14: Performance metrics of the torque actuation matrix $\mathcal{A}_{\mathrm{t}}$ : (a) the manipulability $w_{n}$ and (d) conditioning number $1 / \kappa$ indexes in $P \in \Omega$ when $M$ is aligned along the $x$-direction; $(\mathrm{b}) \Gamma_{w_{n}}\left(\mathcal{A}_{\mathbf{t}}\right)$ and $(\mathrm{e}) \Gamma_{1 / \kappa}\left(\mathcal{A}_{\mathbf{t}}\right)$ for the sampled orientation of $M$; and (c) $\Gamma_{w_{n}}\left(\mathcal{A}_{\mathbf{t}}\right)$ and $\left[(\mathrm{f}) \Gamma_{1 / \kappa}\left(\mathcal{A}_{\mathbf{t}}\right)\right.$ as function of $d_{w}$.

The metrics of the magnetic field gradient are illustrated in Fig. 12. The average of magnetic field gradient behaves equivalently to flat eightelectromagnet configuration. However, as the mag- 
netic field becomes more uniform, its gradient is obviously lower. Next, it appears that the uniformity of the magnetic gradient is decreasing significantly with $d_{w}$, especially, for the component $\frac{\partial b_{y}}{\partial y}$ and $\frac{\partial b_{z}}{\partial z}$.

The performance metrics of the force and torque actuation matrices in the workspace for the sampled orientations of dipole moment $M$ are presented in Fig. 13 and 14 respectively. The manipulability indexes $w_{n}$ of the force and torque have better performance when the orientation of the microrobot magnetic moment $M$ is in the $x y$-plane $\left(\theta=90^{\circ}\right)$ and along $z$-axis $\left(\theta=0^{\circ}\right)$. The conditioning indexes $1 / \kappa$ of force and torque reach a high value when the dipole moment $M$ is aligned along the $z$ global performance metrics of the both force and torque control are increasing with $d_{w}$ in the given OctoMag configuration. Furthermore, the impact of changing the shifting angles of EMA system will be estimated hereafter.

\subsection{Analysis with respect to the tilted angle $\beta$ of electromagnets for EMA systems}

Let us highlight the orientation angle $\beta$ of electromagnets and fix other parameters. Once again, eight electromagnets are applied to enable reliable combined torque and force singular-free control with 5 DOFs. As it has been shown above that long distance $d_{w}$ from the workspace center $O$ to the electromagnet leads globally to a slight more uniform magnetic field and gradient, in addition, the effectiveness control of magnetic microrobot is improved. Nevertheless, stronger magnetic field and gradient require a short length $d_{w}$. With a comprehensive consideration of strength and uniformity, we have set the working distance to $d_{w}=65 \mathrm{~mm}$ that is similar to the OctoMag EMA setup [43, and a workspace of $\Omega=45 \mathrm{~mm} \times 45 \mathrm{~mm} \times 45 \mathrm{~mm}$ is considered throughout the simulations. For the sake of simplicity, the eight electromagnetic coils of the EMA platform are divided in two sets: four stationary electromagnets: $e=1 . .4$; and four mobile coils: $e=5 . .8$, as illustrated in Fig. 8. These two sets are arranged around a common axis of rotation with an azimuth angle $\alpha_{e}=45^{\circ}(e=1 . .8)$, and are common center $O$ of the workspace. The mobile sets are able to rotate their polar angle $\beta_{e} \in\left[0 ; 90^{\circ}\right)(e=5 . .8)$. Two cases are considered for the stationary coils set where the polar $\beta_{e}$ $(e=1 . .4)$ is fixed to:

1. $\beta_{e}=0^{\circ}$, that is equivalent to the OctoMag 43 configuration;
2. $\beta_{e}=26^{\circ}$, that is close to the MiniMag 44 arrangement.

Hence, the considered reconfigurable EMA platform can vary from one to another of these configurations by changing the orientation of the polar angle $\beta_{e}$ in their two sets of electromagnets.

In the following sections, the influence of the mobile angle $\beta_{e}$ on the magnetic field and gradient performance indexes, and on the magnetic actuation indexes are investigated. As previously stated, each electromagnet is computed with a point dipole moment magnitude of: $\left\|M_{e}\right\|=8.178 \mathrm{~A} \mathrm{~m}^{2}$, and the microrobot is modeled from its magnetic moment $M=(1, \theta, \varphi)$, with a unit magnitude, azimuth $\varphi \in\left[0 ; 360^{\circ}\right)$ and polar angles $\theta \in\left[0 ; 180^{\circ}\right]$. Let us recall that the possible collision of the coils is not taken into account in this study.

\subsubsection{Case \#1: reconfigurable OctoMag-like setup}

This section investigates a reconfigurable EMA platform similar to the OctoMag system. Specifically, the mobile set of coils is able to rotate with the same angle $\beta=\beta_{e} \in\left[0 ; 90^{\circ}\right)(e=5 . .8)$. To analyze the performance of such reconfigurable EMA setup, different simulations are conducted, and their results are presented hereafter.

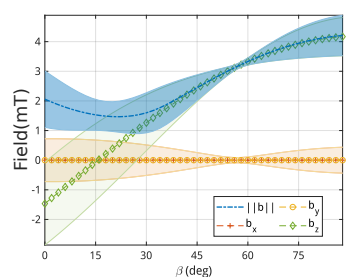

(a)

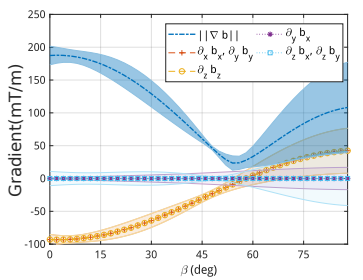

(c)

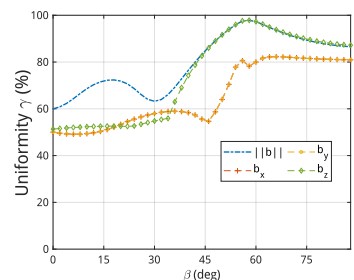

(b)

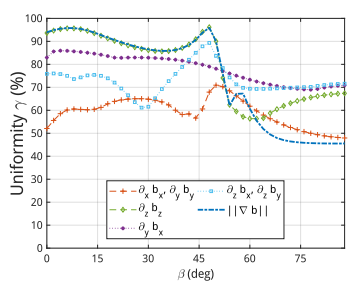

(d)
Figure 15: Performance metrics of (a) (b) the magnetic field and (c) (d) its gradient of reconfigurable OctoMag-like setup when the mobile coils set is rotating with $\beta \in\left[0 ; 90^{\circ}\right)$. The markers and envelopes (a) (c) refer to the mean and standard deviation (STD) of the fields. 

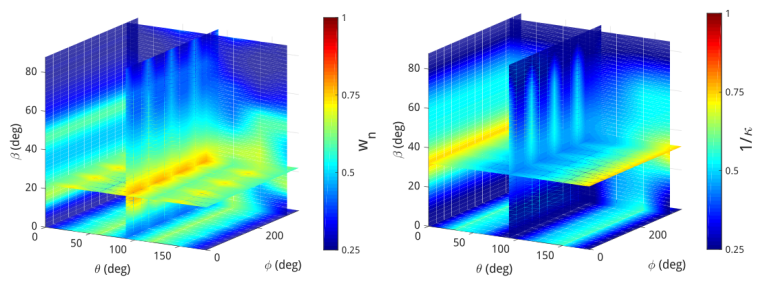

(a) Performance metrics of the(b) Performance metrics of the force $\mathcal{A}_{\mathbf{f}}$ force $\mathcal{A}_{\mathbf{f}}$
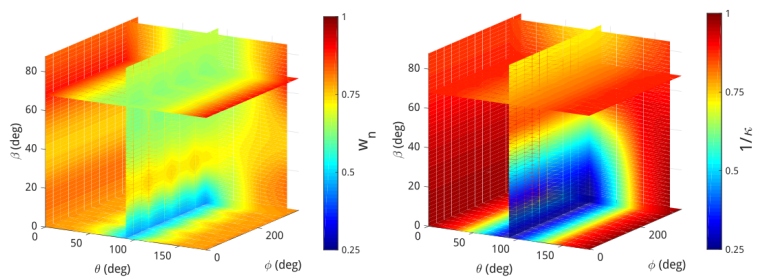

(c) Performance metrics of the(d) Performance metrics of the torque $\mathcal{A}_{\mathrm{t}}$

torque $\mathcal{A}_{\mathrm{t}}$

Figure 16: Actuation performance indexes of the (a) (b) force and (c) (d) torque actuation matrices for the sampled orientations of the magnetic moment of the microrobot: (a) (c) the global normalized manipulability index $\Gamma_{w_{n}}$; and (b). (d) the global conditioning index $\Gamma_{1 / \kappa}$.

First, the magnetic field performance indexes are analyzed and shown in Fig. 15, for different moving angles $\beta \in\left[0 ; 90^{\circ}\right)$. As previously analyzed, the performance indexes are axisymmetric around the $z$-axis, and the magnetic field and gradient behave similarly between the $x$ and $y$ components. In particular, the average values $\left\langle b_{x}\right\rangle$ and $\left\langle b_{x}\right\rangle$ of $B$, and $\left\langle\frac{\partial b_{x}}{\partial y}\right\rangle,\left\langle\frac{\partial b_{x}}{\partial z}\right\rangle$ and $\left\langle\frac{\partial b_{y}}{\partial z}\right\rangle$ of $\nabla B$ are close to zero. Moreover, from these results, two distinguishing be- 80 haviors appear: i) for low angle $\beta \lesssim 45^{\circ}$, the magnetic field gradient is the strongest and more uniform; whereas ii) for higher value of $\beta$, the magnetic field becomes stronger and more uniform. Thus, the reconfigurable OctoMag-like platform exhibits 805 a versatility to manipulate either the magnetic field or its gradient, which is only driven by simply regulating the orientation $\beta_{e}$ of some electromagnets.

Table 4: Minimum and Maximum values of the global actuation performance indexes.

\begin{tabular}{lclll} 
& $\min$ & $(\varphi, \theta, \beta)$ & $\max$ & $(\varphi, \theta, \beta)$ \\
\hline$\Gamma_{w_{n}}\left(\mathcal{A}_{\mathbf{f}}\right)$ & 0.241 & $(0,0,89)$ & 0.772 & $(0,0,22)$ \\
$\Gamma_{1 / \kappa}\left(\mathcal{A}_{\mathbf{f}}\right)$ & 0.133 & $(180,180,89)$ & 0.739 & $(0,0,32)$ \\
\hline$\Gamma_{w_{n}}\left(\mathcal{A}_{\mathbf{t}}\right)$ & 0.446 & $(90,90,0)$ & 0.889 & $(0,0,0)$ \\
$\Gamma_{1 / \kappa}\left(\mathcal{A}_{\mathbf{t}}\right)$ & 0.227 & $(210,90,0)$ & 0.973 & $(0,0,68)$
\end{tabular}

Fig. 16 shows the global performance indexes for the sampled orientations of the magnetic moment $M$ with different moving $\beta$ angles. The simulation results release that if $M$ is parallel to the $x y$-plane (ie. $\theta=90^{\circ}$ ), the global performance indexes of the torque $\mathbf{t}$ are low, whereas the force $\mathbf{f}$ is low when $M$ is aligned along the $z$-axis $\left(\theta=0^{\circ}\right.$ or $\left.180^{\circ}\right)$. To further investigate these results, table 4 reports the maximal and minimal values of the global performance indexes, and the corresponding angles for the sampled orientations of a unit-strength magnetic moment. It can be shown that to improve the perfromacnes of magnetic actuation, firstly, $M$ should be globally aligned along the $z$-axis. Secondly, the moving angle must be set to $\beta=32^{\circ}$ in order to maximize the dexterity index $1 / \kappa$ of the force, whereas a value around $\beta=68^{\circ}$ is required for the torque. In contrast, the normalized manipulability of the force and torque needs a low angle below $\beta \leq 22^{\circ}$ for improvement.

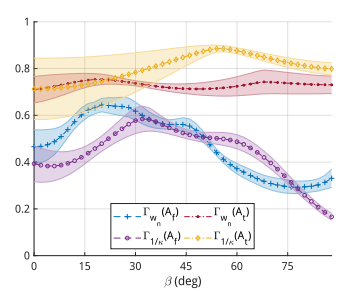

(a)

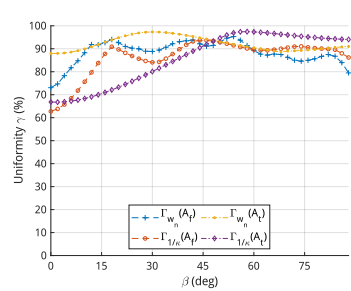

(b)
Figure 17: Statistical data of the global performance indexes of the force and torque actuation matrices for $\beta \in\left[0 ; 90^{\circ}\right)$ : (a) the mean and STD, and (b) the uniformity $\gamma$ metrics.

To exhibit the influence of the moving angle $\beta$ on the magnetic actuation performance, Fig. 17 depicts the mean and uniformity metrics of the global performance indexes $\Gamma_{w_{n}}$ and $\Gamma_{1 / \kappa}$. The analysis of the performance indexes reveals that it is more efficient to control $\mathbf{f}$ with low angle, whereas $\mathbf{t}$ requires a higher $\beta$, especially to increase its uniformity. These simulation results demonstrate that rotating the moving angle $\beta$ could make the EMA platform more flexible to control efficiently either the force $\mathbf{f}$ or the torque $\mathbf{t}$.

Finally, the impact of the moving angle $\beta$ on the workspace size has been also investigated. Since the indexes are axisymmetric around the $z$-axis, the analysis is reported only along this direction. In particular, to evaluate the tissue penetration, the locations behind the workspace $\Omega$ could be considered. The different performance metrics are shown 


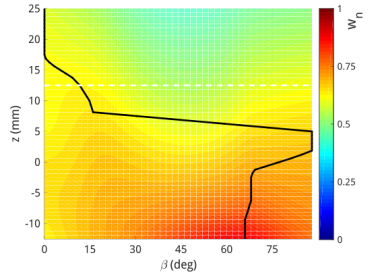

(a)

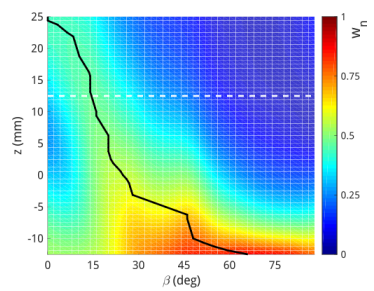

(c)

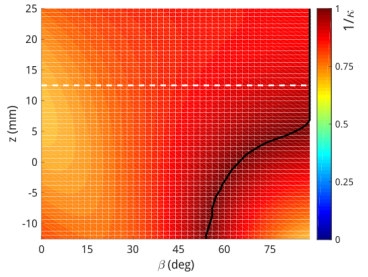

(b)

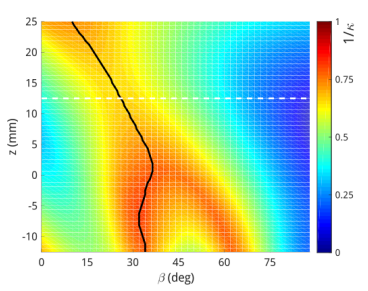

(d)
Figure 18: Performance indexes along the $z$-axis of (a) (b) the torque and (c) (d) the force actuation matrices: (a) (c) the normalized manipulability index $w_{n}$; and (b) (d) the dexterity index $1 / \kappa$. The black line represents the maximum value of the performance indexes.

in Fig. 18 Fig. $18 \mathrm{~b}$ shows that high angle $\beta$ leads to the reliable torque dexterity. Specifically, a high angle $\beta \gtrsim 50^{\circ}$ allows the increasing of the value of ${ }_{845}$ the conditioning number $1 / \kappa$. The good conditioning number of torque means that a reconfigurable EMA system is able to transmit a torque $\mathbf{t}$ along any directions more efficient. However, to enable sufficient force $\mathbf{f}$ in the high locations together with 850 a good manipulability index $w_{n}$, a low angle $\beta \lesssim 30^{\circ}$ is required. Therefore, these results exhibit that to design a versatile EMA system with both effective force and torque control, it is necessary to be able to vary the moving angle $\beta_{e}$ of some coils. Such design objective seems a promising way to achieve 855 optimal control of $\mathbf{f}$ and $\mathbf{t}$.

\subsubsection{Case \#2: reconfigurable MiniMag-like setup}

The second case corresponds to EMA platform, similar to the MiniMag system. Specifically, the mobile set of coils is able to rotate with the same angle $\beta=\beta_{e} \in\left[0 ; 90^{\circ}\right)(e=5 . .8$, as shown in Fig. 8), while the stationary set of coils is fixed to $\beta_{e}=26^{\circ}$ $(e=1 . .4)$.

Fig. 19 shows the performance metrics of the magnetic field and its gradient with a moving angle $\beta \in\left[0 ; 90^{\circ}\right)$. Similarly, the high moving angle value $\beta \gtrsim 45^{\circ}$ provides stronger and more uniform

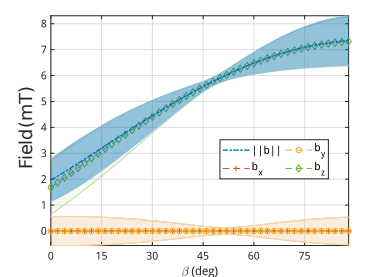

(a)

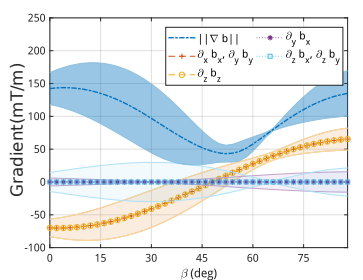

(c)

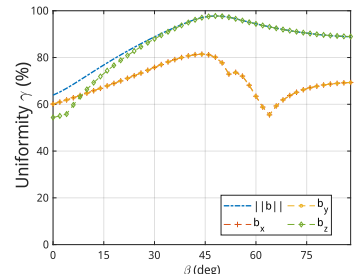

(b)

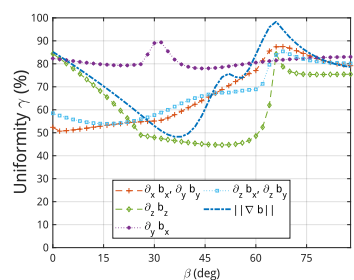

(d)
Figure 19: Performance metrics of (a) (b) the magnetic field and (c) (d) its gradient of reconfigurable MiniMag-like setup when the mobile coils set is rotating with $\beta \in\left[0 ; 90^{\circ}\right)$. The markers and envelopes (a).(c) refer to the mean and standard deviation (STD) of the fields.

magnetic field $B$. Especially, the reconfigurable MiniMag-like setup induces a larger magnetic field strength along the $z$-axis than the OctoMag-like one (cf. Fig. 15). However, the magnetic field gradient requires a low moving angle $\beta$ to be stronger and more uniform. In particular, it can be seen in Fig. 19d that their uniformity metrics $\gamma$ are decreasing for $\beta<26^{\circ}$, and increasing again for $\beta>56^{\circ}$. In such case $\# 2$, it appears that it is more convenient to use high angles around $\beta \approx 50^{\circ}-60^{\circ}$ to get a suitable uniform magnetic field and gradient.

The performance indexes of the actuation matrix $\mathcal{A}(P, M)$ are evaluated for different moving $\beta$ angles, and reported in Fig. 20. From these simulation results, it appears that if $M$ is parallel to the $x y$-plane (ie. $\theta=90^{\circ}$ ), the global conditioning indexes $\Gamma_{1 / \kappa}$ of both the force and torque are low, whereas the global normalized manipulability indexes $\Gamma_{w_{n}}$ are advantageous. Moreover, when $M$ is aligned along the $z$-axis $\left(\theta=0^{\circ} \bmod 180^{\circ}\right)$, a good $\Gamma_{w_{n}}$ is obtained and $\Gamma_{1 / \kappa}$ has the greatest value. Next, the moving angle $\beta$ should have a low value to enable the good global performance indexes $\Gamma_{w_{n}}$ of the torque and the force, while their $\Gamma_{1 / \kappa}$ require a value around $\beta=60^{\circ}$. These trends are clearly revealed in Fig. 21 that shows the magnetic actuation performance indexes statistical data and uniformity 


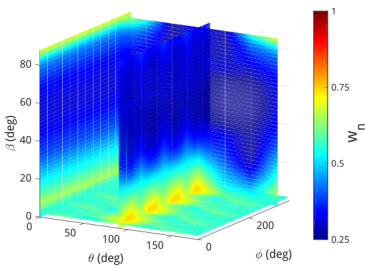
the force $\mathcal{A}_{\mathbf{f}}$

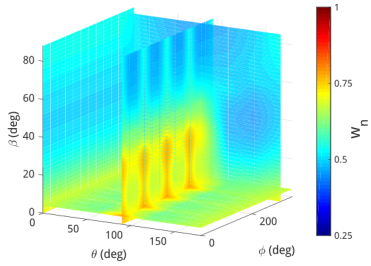

(c) Performance metrics of (a) Performance metrics of the torque $\mathcal{A}_{\mathbf{t}}$

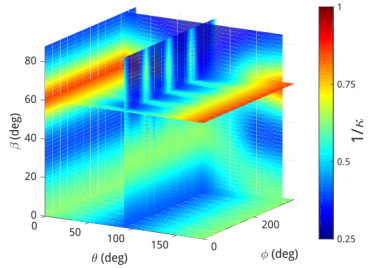

(b) Performance metrics of the force $\mathcal{A}_{\mathrm{f}}$

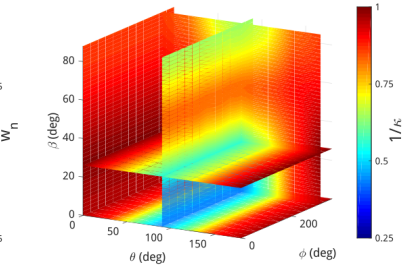

(d) Performance metrics of the torque $\mathcal{A}_{\mathbf{t}}$
Figure 20: Actuation performance indexes of the (a) (b) force and (c) (d) torque actuation matrices for the sampled orientations of the magnetic moment of the microrobot: (a) (c) the global normalized manipulability index $\Gamma_{w_{n}}$; and (b) (d) the global conditioning index $\Gamma_{1 / \kappa}$.

The influence of $\beta \in\left[0 ; 90^{\circ}\right)$ along the $z$-axis has been evaluated and reported in Fig. 22. To maximize the performance indexes, the moving angle should be adjusted with respect to the $z$-depth posiof the magnetic moment $M$ of the microrobot. For instance, Fig. 22d shows that there are two angle routes to provide a force with a good dexterity: one with $\beta \lesssim 35^{\circ}$ and the second with $\beta \gtrsim 65^{\circ}$. Basically, for deep location, a low angle $\beta$ enables a reliable manipulability $w_{n}$ and conditioning number $1 / \kappa$ metrics of both the force and torque. There-

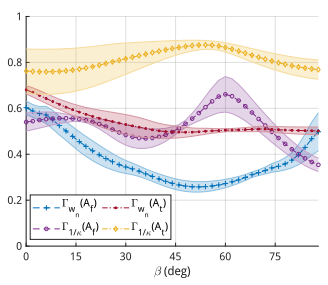

(a)

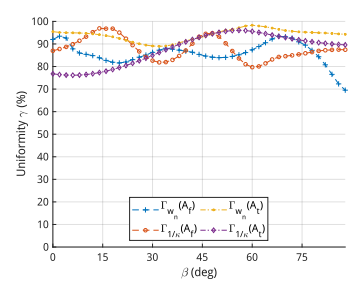

(b)
Figure 21: Statistical data of the global performance indexes of the force and torque actuation matrices for $\beta \in\left[0 ; 90^{\circ}\right)$ : (a) the mean and STD, and (b) the uniformity $\gamma$ metrics. fore, the tilted angle of the applied electromagnet can be determined referring the above analysis for different applications.

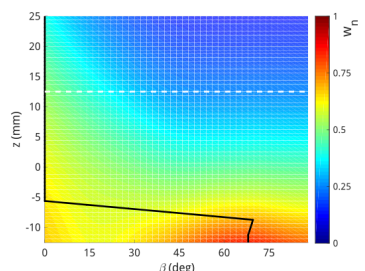

(a)

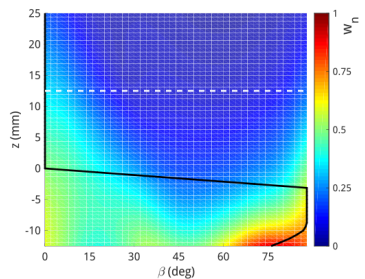

(c)

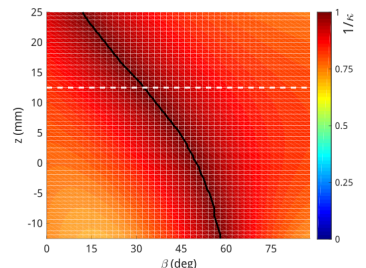

(b)

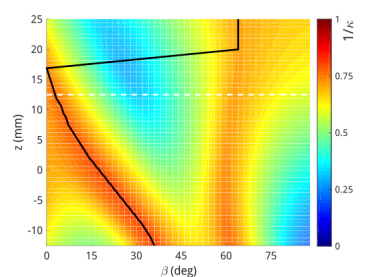

(d)
Figure 22: Performance indexes along the $z$-axis of (a) (b) torque and (c) (d) the force actuation matrices: (c) (a) the normalized manipulability index $w_{n}$; and (d).(b) the dexterity index $1 / \kappa$. The black line represents the maximum value of the performance indexes.

\subsection{Discussions}

Based on the numerous simulations, it appears that MiniMag-like arrangement provides the greatest magnetic field and gradient, besides, the most effective actuation performance can also be performed among the various considered configurations. However, the magnetic field distribution uniformity index Iso of MiniMag-like arrangement is less interesting than OctoMag-like arrangement, implying some difficulties to derive the control strategy. The system is capable of providing different performances by setting the positions and orientations of electromagnets. Therefore, the choice of the basic EMA configuration can be motivated based on the applications objectives and the desired 900 manipulation tasks.

\section{Design analysis}

Different configurations of electromagnetic coils can produce various magnetic field distributions. The flat multi-electromagnet EMA systems are 
used for 2D manipulation. For the 3D control of microrobot, the 3D configurations are applied. In this study, the six different typical systems consisting of flat four-electromagnet, flat six-electromagnet, flat eight-electromagnet, 3D six-electromagnet, OctoMag and MiniMag configurations were simulated and compared. Based on these results, an optimal configuration can be determined for the considered application.

Obviously, the investigated EMA systems all can be reconfigurable where either the distance $d_{w}$ or mobile angle $\beta$ is adjusted. If either the strong magnetic field or gradient is required in 2D plane, $n=2$ and 3 coils are necessary, respectively. The flat four-electromagnet system with short distance $d_{w}$ can be applied for this control with redundancy. Commonly, if 2D applications require only a weak magnetic field and its gradient, a minimum of $n=5$ electromagnets is essential. Thus, the flat sixelectromagnet or flat eight-electromagnet systems set to long distance $d_{w}$ can be used to improve the field distribution and the control redundancy.

Similarly, the 3D EMA systems are also formulated with different distances $d_{w}$ to achieve the desired performances. Especially, the efficiency of OctoMag-like and MiniMag-like systems has been further investigated with respect to the mobile angle $\beta$. When a strong magnetic field and gradient are of prime importance for the application, $d_{w}$ has to be set to a short distance. Moreover, to enhance the magnetic field $\mathrm{B}$, the tilted angle $\beta$ has to be set to an high value. In contrast, to enable a proper magnetic field gradient $\Delta \mathrm{B}$, different specific values of $\beta$ can be considered. Conversely, the long distance $d_{w}$ leads to a more uniform magnetic field and gradient, higher manipulability of torque 900 and force, and higher dexterity of torque and force. Moreover, these performance's improvements can also be realized by implementing the corresponding tilted angles $\beta$ to superimpose their advantages with $d_{w}$

From these results, various system performances can be obtained, depending on the given design parameters. All these capabilities are summarized in the chart given in Fig. 23 and 24. In Fig 23 it is observed that the more electromagnets lead to the 970 stronger magnetic field strength $\langle\|B\|\rangle$. However, the uniformity of field does not change significant by applying more coils. If the magnetic gradient

\footnotetext{
${ }^{1}$ For the sake of consistency between the different results, 975 the magnetic field strength and gradient are here normalized.
}

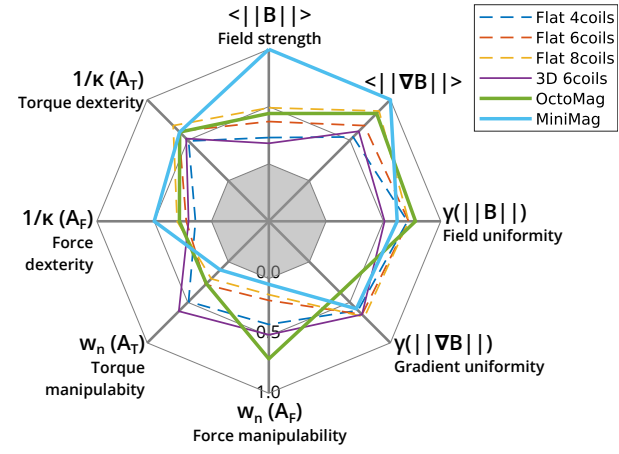

(a)

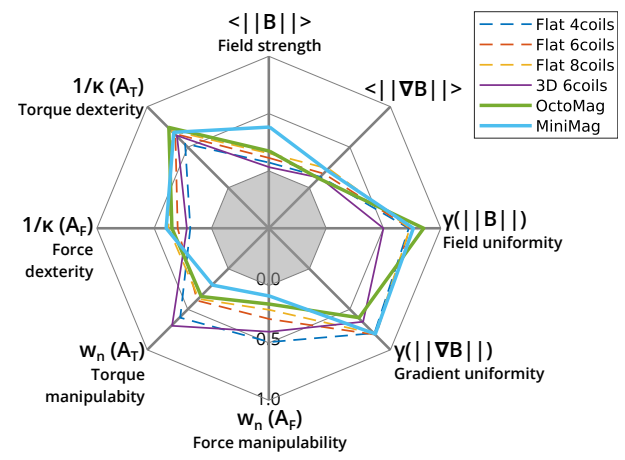

(b)

Figure 23: The optimal performances for each EMA system design for (a) short and (b) long distance $d_{w}$.

is prioritized for control, MiniMag, OctoMag and flat eight-electromagnet configurations are set to short working distance $d_{w}$. Meanwhile, MiniMag, flat eight-electromagnet and 3D six-electromagnet configurations are proposed for long distance $d_{w}$. Considering the uniformity of magnetic field and gradient, MiniMag system has the capability of generating both uniform field and gradient. OctoMag system can produce more uniform magnetic field as well, while its weakness lies in the uniformity of magnetic gradient. Besides, the flat configurations are also able to provide uniform magnetic gradient. The 3D six-electromagnet and flat fourelectromagnet configurations carry the benefit of the good manipulability of torque. Especially in long distance $d_{w}$, flat four-electromagnet arrangement has significant capability of producing better manipulability of force than other configurations. With short distance $d_{w}$, OctoMag system can be used to provide good manipulability of the force. Whereas, the great dexterity of force is produced by using MiniMag system since the eight coils with such configuration could lead to more dexterous 
force control. Such improved phenomenon also oc- 1010 curs in the term of the dexterity of torque through applying more coils.

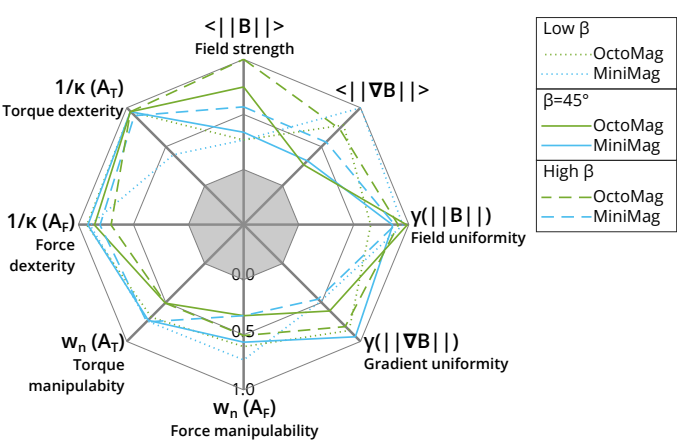

Figure 24: The optimal performances for the OctoMag-like 1025 and MiniMag-like designs regrading the mobile angle $\beta$ value.

The investigation of mobile angle $\beta$ is further represented in Fig. 24. It can be observed that the magnetic field generated by MiniMag-like arrange- 1030 ment becomes much more strong than OctoMaglike configuration when the $\beta$ is set to a high angle value. Conversely, the lower mobile angle allows OctoMag-like system to produce a stronger and more uniform magnetic gradient $\Delta \mathrm{B}$. Comparing ${ }^{1035}$ in Fig. 24. MiniMag-like configuration commonly presents better performance of force and torque control. When $\beta$ is set to around $45^{\circ}$, MiniMaglike configuration has the significant capability to provide more manipulabilities of force and torque. 1040 When $\beta$ is rotated to higher angle, OctoMag-like systems is able to achieve high manipulability of torque control, whereas MiniMag is good at controlling force. Therefore, for the desired application and required system performance, the optimal 1045 configuration or the design inspiration could be obtained from them.

\section{CONCLUSION}

In the paper, theoretical foundation of electromagnetism has been studied, especially, the pointdipole model was analyzed for magnetic field simulation. Moreover, the electromagnetic manipulation of the untethered microrobot was investigated where the motion includes translation and rotation actuated by magnetic force and torque, respectively. Furthermore, the required minimum electromagnetic coils for EMA system were estimated mathematically. Indeed, the linear dependencies regarding the applied field and gradient, and their resulting singular cases have been fully evaluated.

The various configurations of EMA systems have been studied and compared. The performance evaluation of the reconfigurable platform was thereby investigated. Several simulations were proceeded, and performance metrics of the actuated system were analyzed under different shifted distance $d_{w}$ and the tilted angle $\beta$ of electromagnets. Results show that the reconfigurable platform of electromagnets enables a variety of local magnetic field distribution. The evaluations of the force, torque, manipulability and the dexterity indexes demonstrate that the reconfigurable system provides more flexibility. Overall, the shorter distance $d_{w}$ can be used to generate the strong magnetic field and gradient, while their uniformities require longer distance $d_{w}$. Moreover, the low angle $\beta$ leads to the more efficient magnetic force control. But the magnetic torque becomes less controllable as it requires an higher angle $\beta$. Thus, when the configuration of EMA system is stationary, the system design parameters can be determined depend on the above results. If the stationary EMA platform is upgraded to a reconfigurable system by enabling the control of the distance $d_{w}$ and angle $\beta$, it will be resourceful to perform various tasks. Basically, for different applications, by using the mathematical approach as we demonstrated for performant electromagnetic micromanipulation platforms, the obtained EMA system could be optimized and improved concerning the specific medical application. These would help to develop more advanced navigation control strategy of biomedical magnetic microrobot for different micromanipulation tasks. In particular, it can be used for improving the convenience of minimally invasive operation.

\section{References}

[1] F. Tendick, S. S. Sastry, R. S. Fearing, M. Cohn, Applications of micromechatronics in minimally invasive surgery, IEEE/ASME Trans. Mechatronics 3 (1) (1998) 34-42.

[2] M. J. Mack, Minimally invasive and robotic surgery, Jama 285 (5) (2001) 568-572.

[3] S. Purkayastha, T. Athanasiou, R. Casula, S. A. Darzi, Robotic surgery: a review, Hospital Medicine 65 (3) (2004) 153-159.

[4] J. Troccaz, R. Bogue, The development of medical microrobots: a review of progress, Industrial Robot: An International Journal (2008).

[5] S. Tsuda, D. Oleynikov, J. Gould, D. Azagury, B. Sandler, M. Hutter, S. Ross, E. Haas, F. Brody, R. Satava, 
[15] D. Samarasekera, J. H. Kaouk, Robotic single port surgery: Current status and future considerations, Indian journal of urology: IJU: journal of the Urological Society of India 30 (3) (2014) 326.

16] S. U. Bae, W. K. Jeong, S. K. Baek, Current status of robotic single-port colonic surgery, The International Journal of Medical Robotics and Computer Assisted Surgery 13 (1) (2017) e1735.

[17] F. Carpi, C. Pappone, Stereotaxis niobeß magnetic navigation system for endocardial catheter ablation and ${ }_{117}$ gastrointestinal capsule endoscopy, Expert review of medical devices 6 (5) (2009) 487-498.

[18] B. J. Nelson, I. K. Kaliakatsos, J. J. Abbott, Microrobots for minimally invasive medicine, Annual review of biomedical engineering 12 (2010) 55-85.

[19] M. Sitti, Voyage of the microrobots, Nature 458 (7242) (2009) 1121-1122.

[20] M. Sitti, H. Ceylan, W. Hu, J. Giltinan, M. Turan, S. Yim, E. Diller, Biomedical applications of untethered mobile milli/microrobots, Proceedings of the IEEE ${ }_{1185}$ 103 (2) (2015) 205-224.

[21] H. Ceylan, J. Giltinan, K. Kozielski, M. Sitti, Mobile microrobots for bioengineering applications, Lab on a Chip 17 (10) (2017) 1705-1724.

[22] T. Honda, K. Arai, K. Ishiyama, Micro swimming 1190 mechanisms propelled by external magnetic fields, IEEE Transactions on Magnetics 32 (5) (1996) 5085-
5087.

[23] Y. Alapan, O. Yasa, O. Schauer, J. Giltinan, A. F. Tabak, V. Sourjik, M. Sitti, Soft erythrocyte-based bacterial microswimmers for cargo delivery, Science Robotics 3 (17) (2018) eaar4423.

[24] A. Barbot, D. Decanini, G. Hwang, The rotation of microrobot simplifies $3 \mathrm{~d}$ control inside microchannels, Scientific reports 8 (1) (2018) 1-9.

[25] R. W. Carlsen, M. R. Edwards, J. Zhuang, C. Pacoret, M. Sitti, Magnetic steering control of multi-cellular biohybrid microswimmers, Lab on a Chip 14 (19) (2014) 3850-3859.

[26] F. Carpi, N. Kastelein, M. Talcott, C. Pappone, Magnetically controllable gastrointestinal steering of video capsules, IEEE Transactions on Biomedical Engineering 58 (2) (2010) 231-234.

[27] E. Diller, M. Sitti, et al., Micro-scale mobile robotics, Foundations and Trends $\mathbf{R}$ in Robotics 2 (3) (2013) $143-259$.

[28] A. Hosney, J. Abdalla, I. S. Amin, N. Hamdi, I. S. Khalil, In vitro validation of clearing clogged vessels using microrobots, in: 2016 6th IEEE International Conference on Biomedical Robotics and Biomechatronics (BioRob), IEEE, 2016, pp. 272-277.

[29] S. Jeon, S. Kim, S. Ha, S. Lee, E. Kim, S. Y. Kim, S. H. Park, J. H. Jeon, S. W. Kim, C. Moon, et al., Magnetically actuated microrobots as a platform for stem cell transplantation, Science Robotics 4 (30) (2019) eaav4317.

[30] V. H. Le, S. Zheng, J. Han, J.-O. Park, et al., Preparation of tumor targeting cell-based microrobots carrying nir light sensitive therapeutics manipulated by electromagnetic actuating system and chemotaxis, Journal of Micro-Bio Robotics 14 (3-4) (2018) 69-77.

[31] J. Li, B. E.-F. de Ávila, W. Gao, L. Zhang, J. Wang, Micro/nanorobots for biomedicine: Delivery, surgery, sensing, and detoxification, Science Robotics 2 (4) (2017).

[32] J. Li, X. Li, T. Luo, R. Wang, C. Liu, S. Chen, D. Li, J. Yue, S.-h. Cheng, D. Sun, Development of a magnetic microrobot for carrying and delivering targeted cells, Science Robotics 3 (19) (2018) eaat8829.

[33] S. Martel, Microrobotics in the vascular network: present status and next challenges, Journal of MicroBio Robotics 8 (1) (2013) 41-52.

[34] J. J. Abbott, E. Diller, A. J. Petruska, Magnetic methods in robotics, Annual Review of Control, Robotics, and Autonomous Systems 3 (2015).

[35] F. Carpi, N. Kastelein, M. Talcott, C. Pappone, Magnetically controllable gastrointestinal steering of video capsules, IEEE Trans. Biomed. Eng. 58 (2) (2011) 231234.

[36] S. Park, K. Cha, J. Park, Development of biomedical microrobot for intravascular therapy, International Journal of Advanced Robotic Systems 7 (1) (2010) 1.

[37] K. B. Yesin, K. Vollmers, B. J. Nelson, Modeling and control of untethered biomicrorobots in a fluidic environment using electromagnetic fields, The International Journal of Robotics Research 25 (5-6) (2006) 527-536.

[38] T. W. Fountain, P. V. Kailat, J. J. Abbott, Wireless control of magnetic helical microrobots using a rotatingpermanent-magnet manipulator, in: 2010 IEEE International Conference on Robotics and Automation, IEEE, 2010, pp. 576-581.

[39] A. W. Mahoney, J. J. Abbott, Five-degree-of-freedom 
manipulation of an untethered magnetic device in fluid using a single permanent magnet with application in stomach capsule endoscopy, The International Journal 1260 of Robotics Research 35 (1-3) (2016) 129-147.

[40] P. Ryan, E. Diller, Magnetic actuation for full dexterity microrobotic control using rotating permanent magnets, IEEE Transactions on Robotics 33 (6) (2017) 1398-1409.

[41] W. Amokrane, K. Belharet, M. Souissi, A. B. Grayeli A. Ferreira, Macro-micromanipulation platform for inner ear drug delivery, Robotics and Autonomous Systems 107 (2018) 10-19.

[42] S. Schuerle, S. Erni, M. Flink, B. E. Kratochvil, B. J. 1270 Nelson, Three-dimensional magnetic manipulation of micro-and nanostructures for applications in life sciences, IEEE transactions on magnetics 49 (1) (2012) 321-330.

[43] M. P. Kummer, J. J. Abbott, B. E. Kratochvil, 1275 R. Borer, A. Sengul, B. J. Nelson, Octomag: An electromagnetic system for 5-dof wireless micromanipulation, IEEE Transactions on Robotics 26 (6) (2010) 10061017.

[44] B. E. Kratochvil, M. P. Kummer, S. Erni, R. Borer, 1280 D. R. Frutiger, S. Schürle, B. J. Nelson, Minimag: a hemispherical electromagnetic system for 5-dof wireless micromanipulation, in: Experimental Robotics, Springer, 2014, pp. 317-329.

[45] S. Jeon, G. Jang, H. Choi, S. Park, Magnetic navigation 1285 system with gradient and uniform saddle coils for the wireless manipulation of micro-robots in human blood vessels, IEEE transactions on magnetics 46 (6) (2010) 1943-1946.

[46] H. Choi, K. Cha, J. Choi, S. Jeong, S. Jeon, G. Jang, J.- 1290 o. Park, S. Park, Ema system with gradient and uniform saddle coils for 3d locomotion of microrobot, Sensors and Actuators A: Physical 163 (1) (2010) 410-417.

[47] A. Pourkand, J. J. Abbott, A critical analysis of eightelectromagnet manipulation systems: The role of elec- 1295 tromagnet configuration on strength, isotropy, and access, IEEE Robotics and Automation Letters 3 (4) (2018) 2957-2962.

[48] D. J. Griffiths, Introduction to Electrodynamics, 4th Edition, Cambridge University Press, 2017.

[49] D. Jiles, Introduction to magnetism and magnetic materials, CRC press, 2015.

[50] E. Diller, J. Giltinan, G. Z. Lum, Z. Ye, M. Sitti, Sixdegree-of-freedom magnetic actuation for wireless microrobotics, The Int. J. Robot. Res. 35 (1-3) (2016) 1305 114-128.

[51] E. Diller, J. Giltinan, P. Jena, M. Sitti, Three dimensional independent control of multiple magnetic microrobots, in: IEEE Int. Conf. on Intel. Robot. and $\mathrm{Au}-$ tom., 2013, pp. 2576-2581.

[52] S. Schuerle, S. Erni, M. Flink, B. E. Kratochvil, B. J. Nelson, Three-dimensional magnetic manipulation of micro-and nanostructures for applications in life sciences, IEEE Trans. Magn. 49 (1) (2013) 321-330.

[53] J. K. Salisbury, J. J. Craig, Articulated hands: Force 1315 control and kinematic issues, The International journal of Robotics research 1 (1) (1982) 4-17.

[54] T. Yoshikawa, Manipulability of robotic mechanisms, The international journal of Robotics Research 4 (2) (1985) 3-9.

[55] S. Kucuk, Z. Bingul, Comparative study of performance indices for fundamental robot manipulators, Rob. Au- ton. Syst. 54 (7) (2006) 567-573.

[56] J.-P. Merlet, Jacobian, manipulability, condition number, and accuracy of parallel robots, J. of Mech. Des. 128 (1) (2006) 199-206.

[57] S. Schürle, B. E. Kratochvil, S. Pané, M. A. Zeeshan, B. J. Nelson, Generating magnetic fields for controlling nanorobots in medical applications, in: Nanorobotics, Springer, 2013, pp. 275-299.

[58] K. Ishiyama, K. Arai, M. Sendoh, A. Yamazaki, Spiral-type micro-machine for medical applications, in: MHS2000. Proceedings of 2000 International Symposium on Micromechatronics and Human Science (Cat. No. 00TH8530), IEEE, 2000, pp. 65-69.

[59] R. Dreyfus, J. Baudry, M. L. Roper, M. Fermigier, H. A. Stone, J. Bibette, Microscopic artificial swimmers, Nature 437 (7060) (2005) 862-865.

[60] M. N. Faddis, W. Blume, J. Finney, A. Hall, J. Rauch, J. Sell, K. T. Bae, M. Talcott, B. Lindsay, Novel, magnetically guided catheter for endocardial mapping and radiofrequency catheter ablation, Circulation 106 (23) (2002) 2980-2985

[61] T. Roberts, W. Hassenzahl, S. Hetts, R. Arenson, Remote control of catheter tip deflection: an opportunity for interventional mri, Magnetic Resonance in Medicine: An Official Journal of the International Society for Magnetic Resonance in Medicine 48 (6) (2002) 1091-1095.

[62] A. J. Petruska, B. J. Nelson, Minimum bounds on the number of electromagnets required for remote magnetic manipulation, IEEE Trans. Robot. 31 (3) (2015) 714722 .

[63] C. R. Thornley, L. N. Pham, J. J. Abbott, Reconsidering six-degree-of-freedom magnetic actuation across scales, IEEE Robotics and Automation Letters 4 (3) (2019) 2325-2332

[64] J. Giltinan, M. Sitti, Simultaneous six-degree-offreedom control of a single-body magnetic microrobot, IEEE Robotics and Automation Letters 4 (2) (2019) 508-514.

[65] E. D. Diller, J. Giltinan, G. Z. Lum, Z. Ye, M. Sitti, Sixdegrees-of-freedom remote actuation of magnetic microrobots., in: Robotics: Science and Systems, 2014.

[66] Z. Nagy, B. J. Nelson, Lagrangian modeling of the magnetization and the magnetic torque on assembled softmagnetic mems devices for fast computation and analysis, IEEE transactions on robotics 28 (4) (2012) 787797.

[67] K. T. Nguyen, M. C. Hoang, G. Go, B. Kang, E. Choi, J.-O. Park, C.-S. Kim, Regularization-based independent control of an external electromagnetic actuator to avoid singularity in the spatial manipulation of a microrobot, Control Engineering Practice 97 (2020) 104340.

[68] D. Wong, E. B. Steager, V. Kumar, Independent control of identical magnetic robots in a plane, IEEE Robotics and Automation Letters 1 (1) (2016) 554-561.

[69] M. Etiévant, A. Bolopion, S. Régnier, N. Andreff, An improved control-oriented modeling of the magnetic field, in: 2019 International Conference on Robotics and Automation (ICRA), IEEE, 2019, pp. 6178-6184.

[70] X. Jing, W. Guo, Modeling and configuration design of electromagnetic actuation coil for a magnetically controlled microrobot, Chinese Journal of Mechanical Engineering 32 (1) (2019) 63. 